ARTICLE

\title{
Tumor evolution selectively inactivates the core microRNA machinery for immune evasion
}

\author{
Tian-Yu Song (1) 1,2,3,6, Min Long1,3,6, Hai-Xin Zhao 4,6, Miao-Wen Zou (1) 1, Hong-Jie Fan, ${ }^{1,5}$ Yang Liu', \\ Chen-Lu Geng1,3, Min-Fang Song1,2,5, Yu-Feng Liu', Jun-Yi Chen', Yu-Lin Yang ${ }^{4}$, Wen-Rong Zhou ${ }^{4}$, \\ Da-Wei Huang ${ }^{4}$, Bo Peng ${ }^{1}$, Zhen-Gang Peng ${ }^{4} \&$ Yong Cang (i] ${ }^{1 凶}$
}

Cancer cells acquire genetic heterogeneity to escape from immune surveillance during tumor evolution, but a systematic approach to distinguish driver from passenger mutations is lacking. Here we investigate the impact of different immune pressure on tumor clonal dynamics and immune evasion mechanism, by combining massive parallel sequencing of immune edited tumors and CRISPR library screens in syngeneic mouse tumor model and coculture system. We find that the core microRNA (miRNA) biogenesis and targeting machinery maintains the sensitivity of cancer cells to PD-1-independent $T$ cell-mediated cytotoxicity. Genetic inactivation of the machinery or re-introduction of ANKRD52 frequent patient mutations dampens the JAK-STAT-interferon- $\gamma$ signaling and antigen presentation in cancer cells, largely by abolishing miR-155-targeted silencing of suppressor of cytokine signaling 1 (SOCS1). Expression of each miRNA machinery component strongly correlates with intratumoral $T$ cell infiltration in nearly all human cancer types. Our data indicate that the evolutionarily conserved miRNA pathway can be exploited by cancer cells to escape from T cell-mediated elimination and immunotherapy.

\footnotetext{
${ }^{1}$ Gene Editing Center, School of Life Science and Technology, ShanghaiTech University, Shanghai, China. ${ }^{2}$ Center for Excellence in Molecular Cell Science, Chinese Academy of Sciences, Shanghai, China. ${ }^{3}$ Life Sciences Institute, Zhejiang University, Hangzhou, Zhejiang, China. ${ }^{4}$ Oncology and Immunology Unit, WuXi Biology, WuXi AppTec (Shanghai) Co, Ltd, Shanghai, China. ${ }^{5}$ University of Chinese Academy of Sciences, 100049 Beijing, China. ${ }^{6}$ These authors

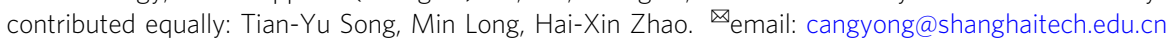


C ancer immunotherapies such as immune checkpoint blockade (ICB) unleash $\mathrm{T}$ cell cytotoxicity against cancer cells and have significantly improved the perspective of cancer patients. However, a majority of cancer patients fail to benefit durably from immunotherapies, mostly due to the cancerintrinsic accumulation of somatic mutations driving primary and acquired resistance to the treatment ${ }^{1-3}$. Two complementary approaches have been adopted to identify genetic elements that control cancer cell sensitivity to T cell-mediated cytotoxicity. One approach is to directly interrogate the genomics and transcriptomics of clinical tumor samples from patients exhibiting variable responses to immunotherapies ${ }^{4-6}$, but the inherent genetic variation between patient cohorts poses a great challenge to pinpoint immunotherapy-relevant drivers from passenger hotspot mutations ${ }^{7,8}$. The other approach is to screen CRISPRCas9-based guide RNA (gRNA) libraries that target either the whole genome using cancer cell and immune cell co-culture systems $s^{9,10}$, or focused gene sets using immunocompetent murine tumors $^{11,12}$. However, in vitro screens are limited to a lack of tumor microenvironment, and the gRNA library coverage or selection often limits the pathological relevance of targets unraveled by in vivo screens.

Combined, these approaches have led to the discovery of a myriad of mechanisms underlying cancer susceptibility to or evasion from $\mathrm{T}$ cell attack. Among them, the most prominent regulators affect antigen presentation and interferon- $\gamma$ $(\mathrm{IFN} \gamma)$ signaling (B2M, JAK1/2, SOCS1, PTPN2, APLNR, and ADAR1) $4,11,13-16$, in addition to chromatin remodeling (PBRM1, ARID2, KMT2D, and ASF1A) $)^{5,9,12,17}$, and TNFa and autophagy pathways ${ }^{10,18}$. Despite these advances, our understanding of the immune evasion mechanism remains incomplete to overcome recurrent clinical resistance. Evolving cancer cells could theoretically accumulate many more mutations that regulate their responses or resistance to tumor immunity ${ }^{7,11}$. It is therefore imperative to uncover novel immune evasion mechanisms in the dynamic tumor-immune microenvironment by untangling the genetic heterogeneity of cancer cells.

MicroRNAs (miRNAs) are small non-coding RNAs that bind complementary messenger RNAs (mRNAs) to repress gene expression and regulate essentially all cellular processes ${ }^{19}$. MiRNAs are generated by stepwise cleavage via double-stranded ribonuclease III enzymes DROSHA and DICER1 and loaded onto Argonaute (AGO) proteins to pair with target sequences on mRNAs ${ }^{20}$. The miRNA-mRNA pairing triggers casein kinase $1 \alpha$ $(\mathrm{CK} 1 \alpha)$-induced phosphorylation and dissociation of AGO2 from the active complex, whereas the ANKRD52-PPP6C phosphatase complex dephosphorylates AGO2 to restore its miRNA loading activity $^{21,22}$. Recurrent mutations in these core miRNA machinery components are identified in many cancer types including neuroblastoma, Wilms tumor, ovarian cancer, and melanoma ${ }^{23-28}$. For example, mutations in or reduced expression of DROSHA and DICER1 in tumors associate with advanced tumor stage and poor clinical outcome in cancer patients ${ }^{29-31}$. Additionally, ANKRD52 was found as a suppressor of tumor metastases, and reduced ANKRD52 levels are associated with late-stage lung cancer ${ }^{22,32}$.

Although mutations in or dysregulation of miRNA machinery characterize a sizeable patient subpopulation and play crucial roles in cancer development, whether these genetic alterations in cancer cells could contribute to immune evasion or resistance to ICB is still unclear. Here, we uncover an unexpected role of the core miRNA machinery in enabling cancer cell sensitivity to $\mathrm{T}$ cell-mediated cytotoxicity by untangling tumor heterogeneity during unbiased immune selection. We demonstrate that disruption of the machinery mitigates miR-155-targeted silencing of SOCS1 in cancer cells, and consequently suppresses essential signaling for $\mathrm{T}$ cell-mediated anti-tumor immunity.

\section{Results}

Profiling of cancer heterogeneity selected by increasing host immunity. Cancer cell lines are genetically unstable and heterogeneous by natural selection ${ }^{33,34}$. We hypothesized that host immune pressure can selectively enrich cells expressing genes acquiring immune-escaping mutations during tumor expansion. To unravel such mutations, we implanted the MC38 murine colorectal cancer (CRC) cell line to C57BL/6 mice (wildtype, WT), and treated them with monoclonal antibodies against mouse programmed death protein 1 (PD-1) or programmed cell death ligand protein 1 (PD-L1). Nude mice were used as a control host for tumor growth under minimum $\mathrm{T}$ cell selective pressure (Supplementary Fig. 1a). Engrafted tumors were dissected and categorized as immunodeficient $(n=10)$, immunocompetent $(n=13)$, and immunotherapy groups $(n=8)$ for deep $(40 \mathrm{M}$ reads of) RNA sequencing (Fig. 1a).

Compared to the immunodeficient group, the expression signature of key immunomodulators and pathways of adaptive immunity ${ }^{35}$ were significantly enhanced in the immunocompetent group and further enhanced in the immunotherapy group, indicating escalating immune pressure (Supplementary Fig. 1b-d). After calling mutations using Genome Analysis Toolkit $(\mathrm{GATK})^{36}$, we identified 59 non-synonymous somatic mutations (24 missense, 18 splicings, 15 frameshifts, 2 deletions) from expressed transcripts ${ }^{34,37}$ that were uniquely present in at least two different immunocompetent tumors, compared with cultured cells or any immunodeficient tumors. Among 53 genes affected by these mutations, 23 exhibited no alteration in any immunotherapy tumor, suggesting that cells with mutations in these genes might be eliminated by $\mathrm{T}$ cells after blockade of PD-1/ PD-L1 checkpoint. The remaining 30 were found in tumors from both immunocompetent and immunotherapy groups, suggesting that they might facilitate cancer escape from $\mathrm{T}$ cell immunity independent of the PD-1 checkpoint (Fig. 1b).

Targeted in vivo CRISPR screen for mutations driving immune evasion. To validate the mutations that drive cancer evasion from $\mathrm{T}$ cells ${ }^{8}$, we created a library composed of singleguide (sg) RNAs targeting these 53 candidate genes (10 sgRNAs per gene) and positive control genes ( $P d c d 1, C d 274$, Jak1, Jak2, and $B 2 m$ ), named as mouse Mutagenesis from Cancer Evolution, or mMCE, library (Supplementary Fig. 2a). This library was introduced to MC38 cells (>1000 × coverage) and implanted to T cell-depleted mice (Nude mice or CD4 and CD8 antibodies treatment), or WT mice with or without PD-1/PD-L1 antibody treatment (Fig. 2a). As expected, loss of T cells accelerated tumor growth, while blocking the PD-1 checkpoint inhibited tumor growth (Fig. 2b; Supplementary Fig. 2b). Tumors grown under these variable immune pressures were collected for amplicon sequencing to determine the sgRNA representation (Supplementary Fig. 2c), which revealed a very high consistency between biological replicate tumors in each group (Supplementary Fig. 2d; Pearson's correlation (COR) $>0.58$ for every two tumors).

MAGeCK and EdgeR analyses of guide abundance revealed that almost all PD-L1 sgRNAs were depleted in tumors from WT mice compared to $\mathrm{T}$ cell-depleted mice, but enriched in immunotherapy tumors compared to WT tumors (Fig. 2c; Supplementary Data 1), consistent with the inhibitory function of PD-L1 in cancer cells to induce exhaustion of PD-1-expressing $\mathrm{T}$ cells $^{38}$. The same distribution was observed for sgRNAs targeting Jak1 and Jak2 (Supplementary Fig. 3a-d; Supplementary Data 1), which mediate the response to anti-PD-1 therapy in melanoma patients ${ }^{39}$. Similar to our results, sgRNAs for key IFN $\gamma$ signaling genes including Jak1/2 have been reported to exhibit different or even opposing performance between in-vivo and in- 
a

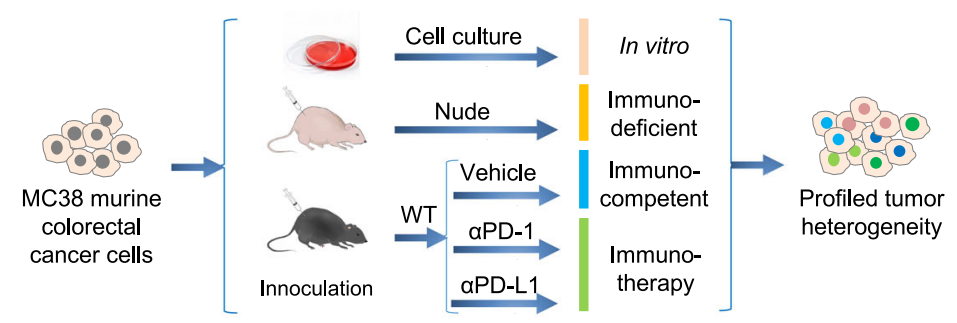

b

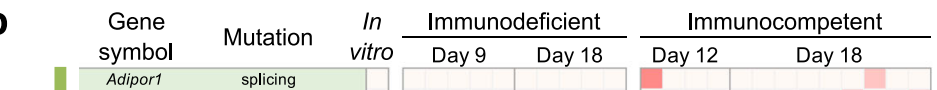

Immunotherapy
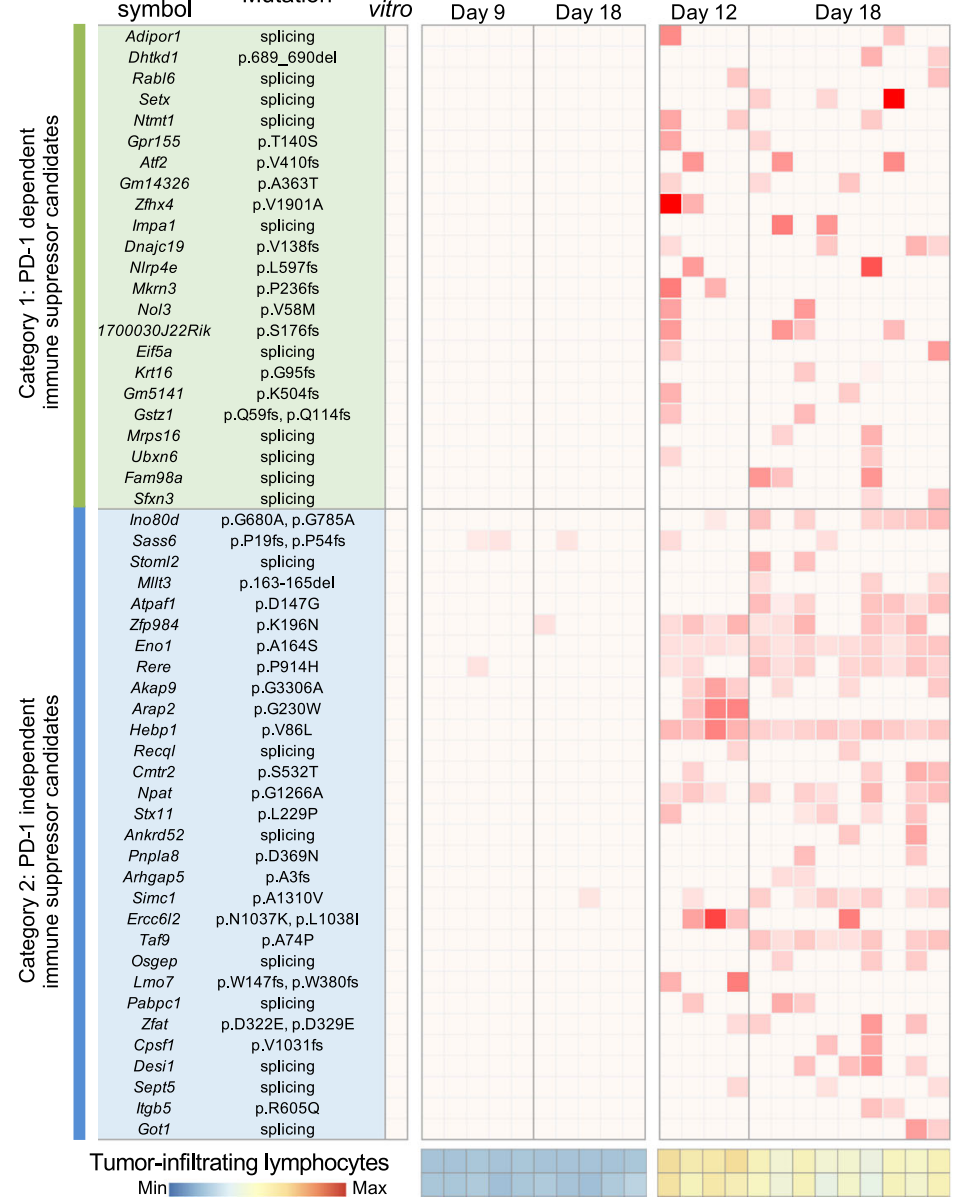

aPD-L1 aPD1

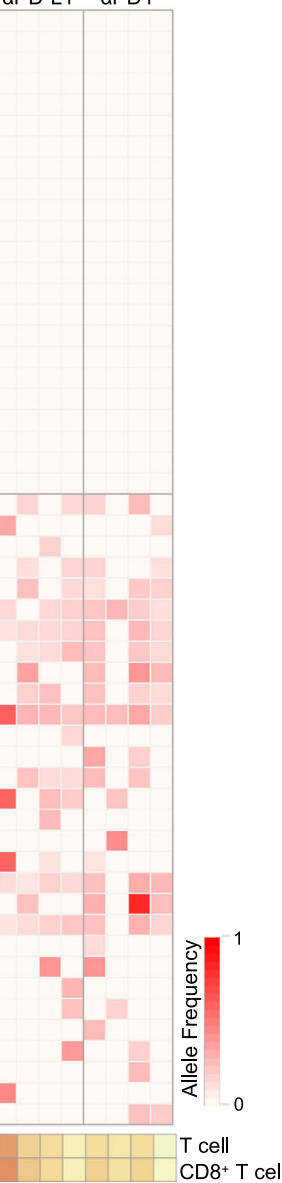

Fig. 1 Clonal mutation profiling of tumors under progressive immune selection. a Schematic overview of clonal mutation profiling of MC38 cell linederived tumors grown in host mice harboring different levels of T cell immunity. $\mathbf{b}$ Heatmap showing allele frequency (AF) of hotspot mutations enriched $(A F>0.1)$ in at least two different tumors from the immunocompetent group ( $n=10$ mice for immunodeficient, $n=13$ for immunocompetent, and $n=8$ for immunotherapy group). A total of 59 mutations in 53 genes were categorized to PD-1-dependent and -independent groups as indicated. Tumor-infiltrating lymphocytes were calculated by mMCP counter. See also Supplementary Fig. 1. Source data are provided as a source data file.

vitro CRISPR library screens ${ }^{10,40}$. Inhibiting IFN $\gamma$ signaling in low/absent MHC-I tumors stimulated the production of IFN $\gamma$ by exhausted $\mathrm{T}$ cells, which drove the maturation of innate immune cells to kill tumor cells ${ }^{41}$. By contrast, sgRNAs targeting PD-1, mainly expressed on activated $\mathrm{T}$ cells but not on tumor cells ${ }^{42}$, did not exhibit obvious abundance changes in tumors across all groups (Fig. 2d). These results corroborate the screen robustness.

Resistance to $\mathrm{T}$ cell immunity by ANKRD52 loss. Furthermore, we found that 6-8 out of 10 sgRNAs targeting Ankrd52 were significantly enriched in WT and immunotherapy tumors compared to $\mathrm{T}$ cell-depleted tumors (Fig. 2e, f), suggesting that inactivation of ANKRD52 conferred a selective advantage for tumor cells against PD-1 independent T cell-mediated immunity. The significance of this distribution pattern is reinforced by the presumably loss-of-function splice-site alteration (NM_172790.2: c.2723-2 A > G) in Ankrd52 that was enriched in both WT and immunotherapy tumors (Fig. 1b). Although alterations of several genes (such as Hebp1, Eno1, and Taf9) other than Ankrd52 are more frequently detected in our tumor profiling, their targeting sgRNAs exhibited much less enrichment or fold-change as compared to those of Ankrd52 (Supplementary Fig. 3e; Supplementary Data 1). We, therefore, selected Ankrd52 for further studies.

To validate the role of Ankrd52 in immune evasion, we first performed in vivo competition assays by infecting MC38 cells individually with four Ankrd52 sgRNAs and three non-target sgRNAs, followed by implanting the mixture of the same number of cells from the 7 pools to WT or nude mice (Supplementary Fig. 4a, b). As expected, each Ankrd52 guide became significantly 
a

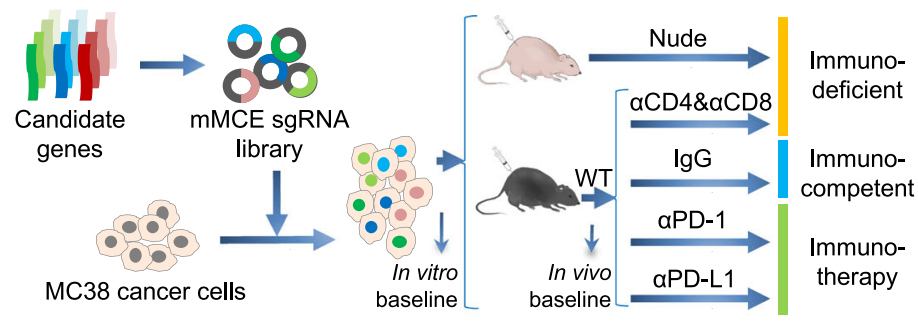

C

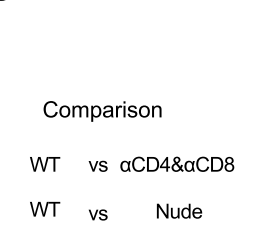

aPD-1 vs $\alpha C D 4 \& \alpha C D 8$

aPD-L1 vs aCD4\&aCD8

aPD-1 vs Nude

aPD-L1 vs Nude

aPD-1 vs WT

aPD-L1 vs WT
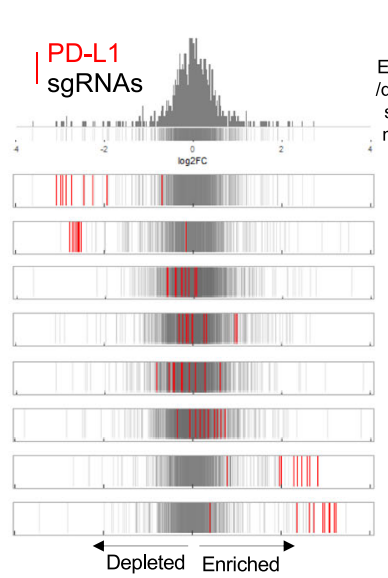

d

10

9

0

2

1

0

10

9 b
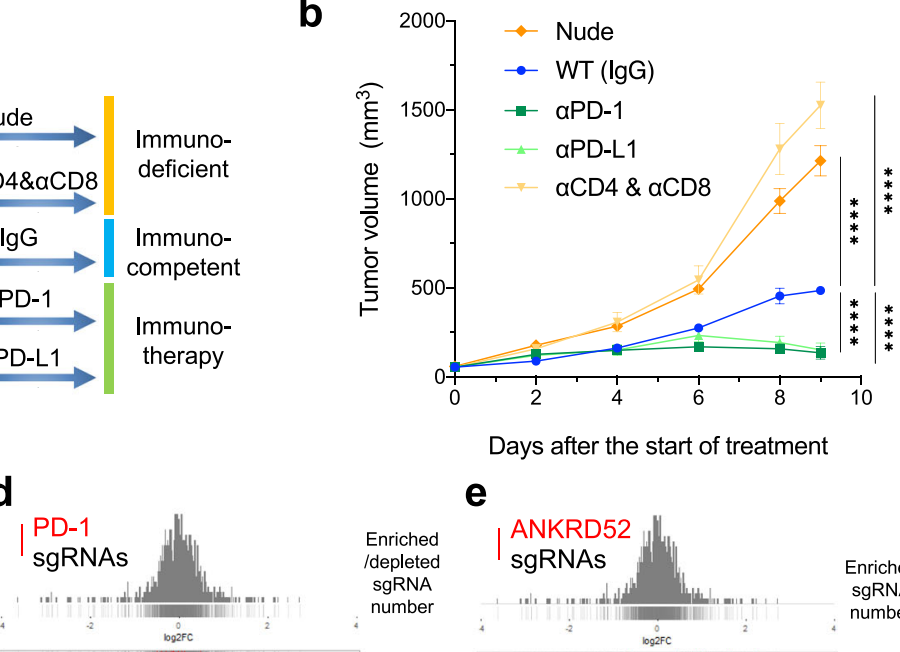

e

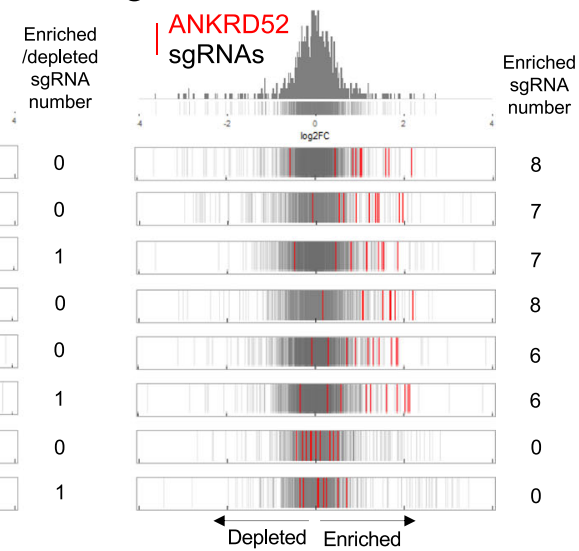

f

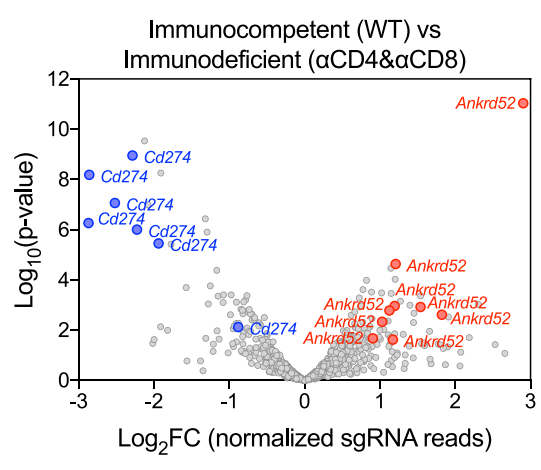

g

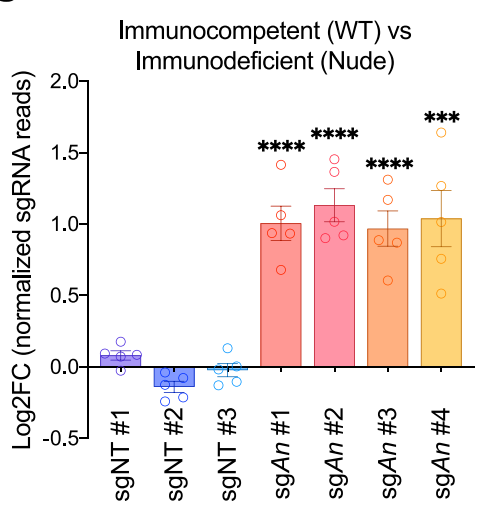

h

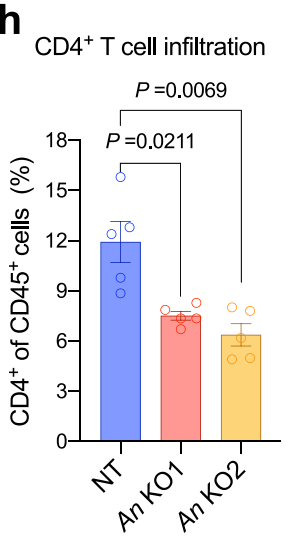

Fig. 2 In vivo CRISPR screen targeting profiled mutations identifies ANKRD52 as a key modulator of cancer immunity. a Schematic overview of in vivo CRISPR screen to validate candidates from immune-selected mutations. b Tumor growth curves of MC38 tumors in nude mice $(n=8)$, and WT mice treated with rat IgG2a and IgG2b isotype $(n=8)$, PD-1 antibody $(n=10)$, PD-L1 antibody $(n=10)$, or CD4 and CD8 antibodies ( $n=7)$. Data are represented as mean \pm s.e.m., ${ }^{\star \star \star \star} P<0.0001$, significance was determined using two-way analysis of variance (ANOVA). c-e Distribution histograms of $\log _{2}$ Fold-change (FC) for all 10 sgRNAs targeting Cd274 (c), Pdcd1 (d) or Ankrd52 (e) as indicated in red lines, overlaid on gray gradient depicting the overall distribution (Cut-off: $|\mathrm{FC}|>1.5, P<0.05$ for enrichment or depletion, analyzed by edgeR). f, Volcano plot for selected top guides for Cd274 and Ankrd52 (Cut-off: $|F C|>1.5, P<0.05$, analyzed by MAGeCK). $\mathbf{g}$ In vivo competition assay with equal number mixture of MC38 cells infected with sgRNA for non-targeting control (NT) or Ankrd52 (An) in WT mice $(n=5)$ and nude mice $(n=5)$. Data are represented as mean \pm s.e.m., ${ }^{\star \star \star} P=0.0004$, ${ }^{\star \star \star \star} P<0.0001$, significance was determined using two-tailed unpaired Student's $t$-test to compare sgAnkrd52 vs sgNT \#2. h, i, Flow cytometry analysis of $\mathrm{CD}^{+}(\mathbf{h})$ and $\mathrm{CD}^{+}(\mathbf{i})$ T cell populations from NT and Ankrd52 knockout ( $\left.A n \mathrm{KO}\right)$ tumors ( $n=5$ per group). Data are representative of two independent experiments and represented as mean \pm s.e.m., significance was determined using two-tailed unpaired Student's t-test. See also Supplementary Fig. 2-5. Source data are provided as a source data file.

enriched in WT tumors compared to nude tumors, indicating growth advantages over control cells (Fig. $2 \mathrm{~g}$ ). Next, we generated Ankrd52-null MC38 cells using CRISPR-Cas9 gene editing and found these cells exhibited growth disadvantage over extended time in long-term culture or as tumors grown in $\mathrm{T}$ cell-depleted mice (Supplementary Fig. 4c, d), a phenomenon supported by
CRISPR library screen results (Supplementary Fig. 4e, f) and likely as a result of p53/p21 activation (Supplementary Fig. 4g). However, the growth disadvantage of Ankrd52-null tumors was abolished when implanted to WT mice (Supplementary Fig. 5a), suggesting that loss of ANKRD52 enabled cancer cells to escape T cell immunity in vivo, thereby offsetting their intrinsic growth 
a

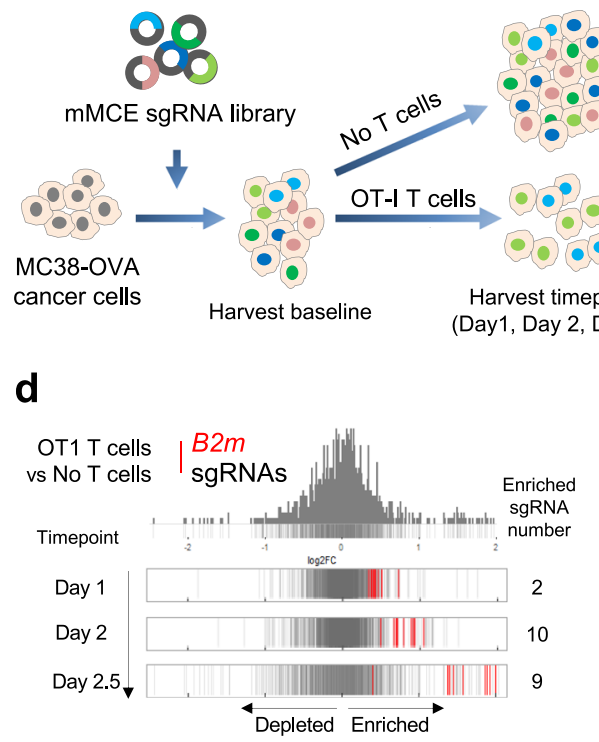

b

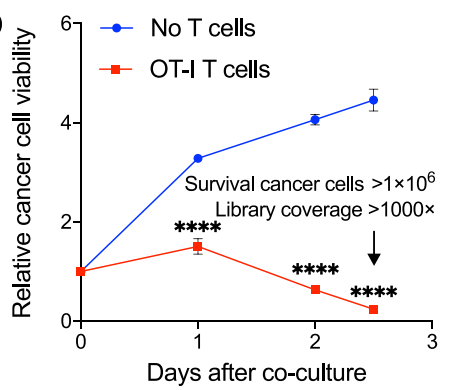

C

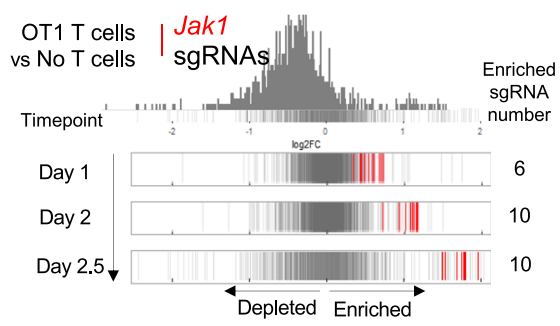

e

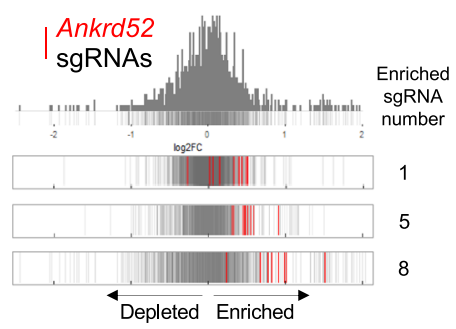

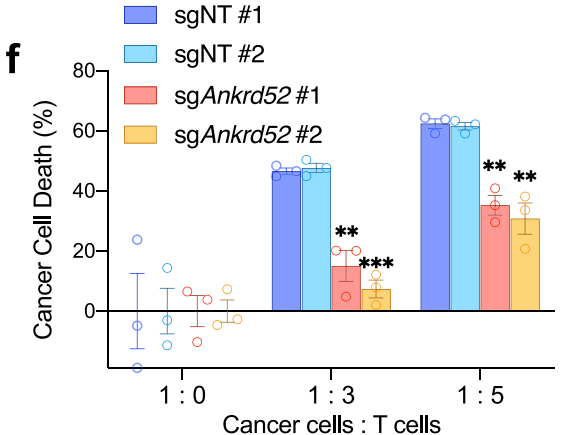

Fig. 3 Targeted in vitro CRISPR screen identifies ANKRD52 as a direct modulator of cancer sensitivity to T cell cytotoxicity. a Schematic overview of CRISPR screen using co-culture of OT-I T cell with MC38-OVA cells. b Cell viability of MC38-OVA cells co-cultured with OT-I T cells during 2.5 days ( $n=3$ per group per timepoint). Data are represented as mean \pm s.d., ${ }^{\star \star \star \star} P<0.0001$, significance is determined using multiple two-tailed Student's $t$-test. c-e Distribution histograms of $\log _{2} \mathrm{FC}$ values for all ten sgRNAs targeting Jak1 (c), B2m (d), or Ankrd52 (e) (Cut-off: FC $>1.4, P<0.05$ for enrichment, analyzed by edgeR). $\mathbf{f}$ Killing of MC38-OVA cells with indicated sgRNAs by OT-I T cells at indicated ratio in co-culture. Data are representative of three independent experiments and represented as mean \pm s.e.m., ${ }^{\star \star} P<0.01,{ }^{\star \star \star} P<0.001$, significance was determined using multiple two-tailed Student's $t$-test (sgNT \#1 vs sgAnkrd52 \#1, $P=3.72 \times 10^{-3}$ for $1: 3, P=1.74 \times 10^{-3}$ for 1:5; sgNT \#2 vs sgAnkrd52 \#1, $P=3.64 \times 10^{-3}$ for $1: 3, P=1.67 \times 10^{-3}$ for $1: 5 ;$ sgNT \#1 vs sgAnkrd52 \#2, $P=2.28 \times 10^{-4}$ for 1:3, $P=4.43 \times 10^{-3}$ for 1:5; sgNT \#2 vs sgAnkrd52 \#2, $P=2.7 \times 10^{-4}$ for 1:3, $P=4.57 \times 10^{-3}$ for 1:5). See also Supplementary Figs. 6 and 7. Source data are provided as a source data file.

deficiency. Tumor-infiltrating lymphocyte (TIL) analysis revealed that the proportions, but not activation or cytotoxicity, of $\mathrm{CD} 4^{+}$ and $\mathrm{CD}^{+} \mathrm{T}$ cells were significantly decreased in Ankrd52-null tumors (Fig. 2h, I; Supplementary Fig. 5b-g). Exhausted T cells $\left(\mathrm{LAG}^{+}{ }^{+} \mathrm{PD}^{+}\right.$or $\mathrm{TIM}^{+}{ }^{+} \mathrm{PD}^{+}$) were also decreased (Supplementary Fig. 5h, i), possibly explaining why Ankrd52 mutation was not eliminated by PD-1 blockade in our tumor mutation profiling (Fig. 1b).

ANKRD52 is a direct modulator for $\mathbf{T}$ cell killing. To further investigate whether ANKRD52 or other immune evasion mutations participate in cancer sensitivity to $\mathrm{T}$ cell-mediated cytotoxicity directly, we performed a parallel in vitro CRISPR screen whereas MC38 cells expressing ovalbumin (MC38-OVA) were introduced with the mMCE sgRNA library and then co-cultured with antigen-specific OT-I T cells (Fig. 3a, b; Supplementary Fig. 6a, b). Like Jak1/2 and B2m, Ankrd52 sgRNAs exhibited time-dependent enrichment in tumor cells in the presence of $\mathrm{T}$ cells (Fig. 3c-e; Supplementary Fig. 6c-g; Supplementary Data 2), providing direct evidence that loss of ANKRD52 conferred resistance to T cell killing. MC38-OVA or OVA peptidetreated MC38 cells with depleted ANKRD52 became less sensitive to the killing by OT-I T cells and suppressed the proliferation of T cells when co-cultured (Fig. 3f; Supplementary Fig. 7). Putting together, CRISPR library screens in vitro and in vivo demonstrate a direct role of ANKRD52 in modulating cancer sensitivity to $\mathrm{T}$ cell-mediated clearance.

Attenuated IFN $\gamma$ response by ANKRD52 loss. We next sought to determine how ANKRD52 alters cancer cell's interaction with $\mathrm{T}$ cell by comparing the transcriptome of Ankrd52-null cells with control cells. Loss of ANKRD52 caused a remarkable downregulation in the expression of IFN $\gamma$ and IFNa responsive gene sets after MC38 cells were stimulated with IFN $\gamma$ (Fig. $4 a-d$; Supplementary Fig. 8a-d; Supplementary Data 3), a T cell cytokine essential for tumor immunity ${ }^{43}$. Among them were PD-L1 and effector $\mathrm{T}$ cell-recruiting chemokines such as CXCL9 and CXCL10 (Fig. 4d) ${ }^{44}$, an observation confirmed by flow cytometry analysis and quantitative PCR (Fig. 4e, f; Supplementary Fig. $9 \mathrm{a}-\mathrm{c})$ and in accordance with the decreased infiltration of $\mathrm{T}$ cells in Ankrd52-null tumors (Fig. 2h, i). Strikingly, IFN $\gamma$ induced expression of TAP1, a key component of the antigen processing machinery, was completely abrogated by ANKRD52 depletion (Fig. 4d, g). Accordingly, significant decrease in total surface MHC-I was observed in both Ankrd52-null MC38 cancer cells isolated from tumors (Supplementary Fig. 9d, e) or cultured with IFN $\gamma$ stimulation (Fig. 4h; Supplementary Fig. 9f). Presentation of the SIINFEKL epitope from OVA in the context of $\mathrm{H} 2 \mathrm{~K}^{\mathrm{b}}$ was also significantly lower in IFN $\gamma$-stimulated ANKRD52deficient cells than control cells (Fig. 4i; Supplementary Fig. 9g, h), suggesting that loss of ANKRD52 decreased the levels of antigen-loaded MHC-I on the surface of tumor cells and thus compromised their recognition by $\mathrm{T}$ cells.

Impaired IFN $\gamma$ response by clinical $A N K R D 52$ mutations. IFN $\gamma$ response is regulated by JAK-STAT signaling 45 . Activation of STAT1 by IFN $\gamma$ was markedly diminished by ANKRD52 depletion (Fig. 4g). Compared with the wildtype gene, four hotspots ANRKD52 mutations (G413W, E506D, S511P and A745T) identified in cancer patient samples (Fig. 5a) failed to effectively rescue the activation defect of STAT1 (Fig. 5b) or upregulation of membrane MHC-I levels (Fig. 5c) upon IFN $\gamma$ treatment, and thus maintained cancer cell resistance to T cell-mediated killing (Fig. $5 \mathrm{~d}$ ). Analysis of several published datasets on cancer patients receiving 
a

a Top gene sets for common downregulated genes

(An KO +IFNY vs NT +IFNy)

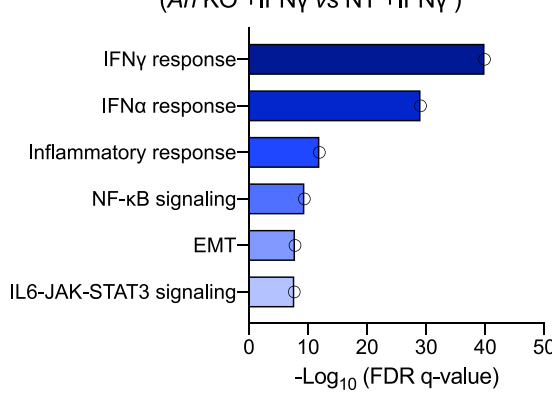

b

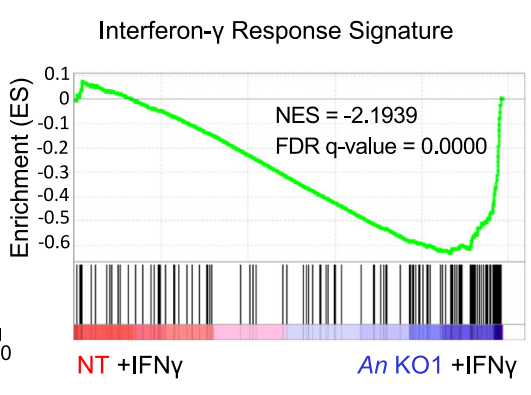

C

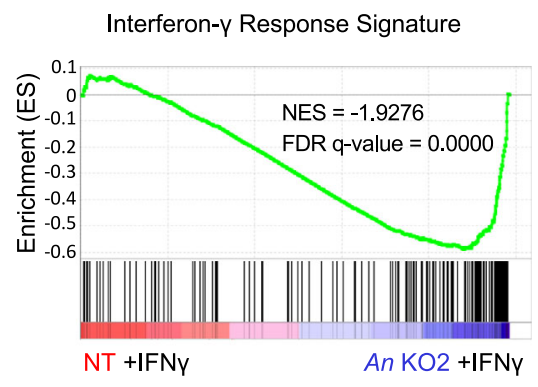

d IFNY
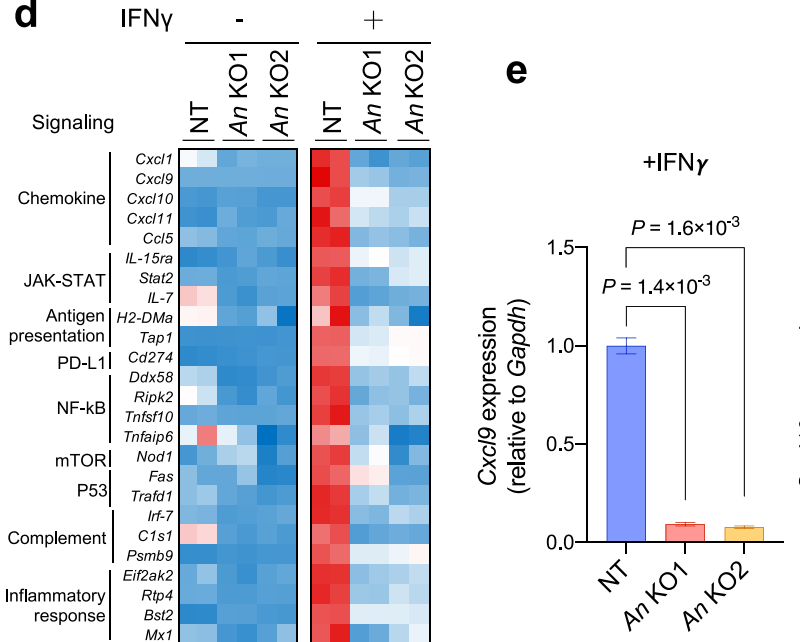

h

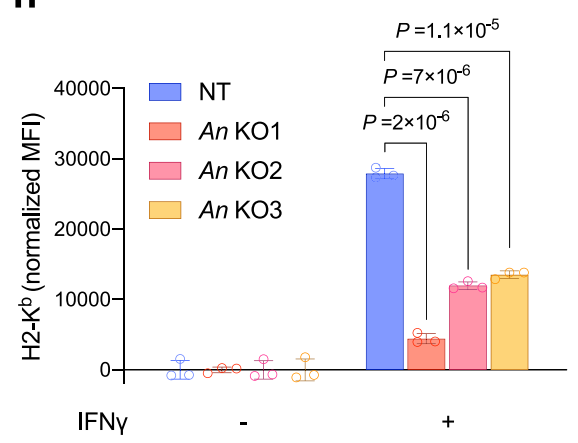

g

$$
\text { IFNY }
$$
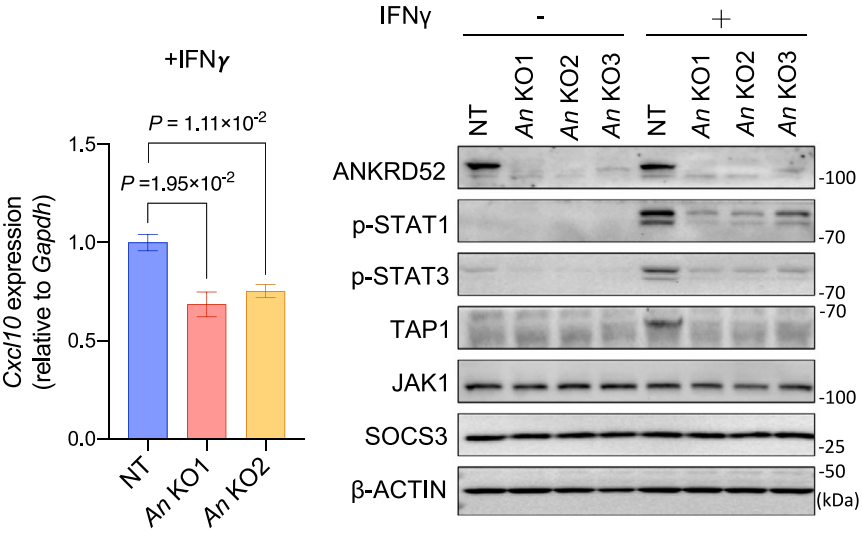

i

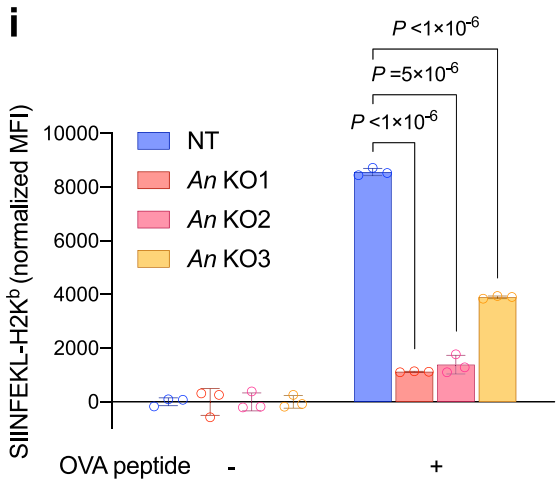

Fig. 4 ANKRD52 inactivation attenuates IFN $\gamma$ signaling, chemokine expression, and antigen presentation. a Hallmark gene sets enriched for commonly down-regulated genes in Ankrd52-null (both $A n \mathrm{KO}$ and $A n \mathrm{KO} 2,1.5$-fold-change cut-off, $P<0.05$, analyzed by edgeR) MC38 cells compared to control cells after IFN $\gamma$ treatment. b, c Enrichment of genes associated with IFN $\gamma$ response in Ankrd52-null cells exposed to IFN $\gamma$. d Heatmap showing downregulated IFN $\gamma$ responsive gene expression in Ankrd52-null cells by RNA-seq analysis ( $n=2$ per group per condition). e, $\mathbf{f} C x c 19$ (e) and Cxcl10 (f) mRNA level in control and ANKRD52-null MC38 cells treated with IFN $(n=3$ per group). Data are representative of two independent experiments and represented as mean \pm s.e.m., significance was determined using two-tailed unpaired Student's $t$-test. $\mathbf{g}$ Abundance of IFN $\gamma$ signaling proteins in control and Ankrd52-null MC38 cells treated with IFN $\gamma$. Data are representative of five independent experiments. $\mathbf{h}$ Abundance of membrane MHC-I expression in control and Ankrd52-null MC38 cells after treatment with IFN $\gamma$. MFI (mean fluorescence intensity) of H2- $\mathrm{K}^{\mathrm{b}}$ was normalized by the responding group without IFN $\gamma$ treatment ( $n=3$ per group per condition). Data are representative of four independent experiments and represented as mean \pm s.d., significance was determined using two-tailed unpaired Student's $t$-test. i Presentation of OVA-derived peptide (SIINFEKL) in OVA-treated control and Ankrd52-null MC38 cells. MFI of SIINFEKL-H2K ${ }^{b}$ was normalized by the responding group without IFN $\gamma$ treatment ( $n=3$ per group per condition). Data are representative of three independent experiments and represented as mean \pm s.d., significance was determined using two-tailed unpaired Student's $t$-test. See also Supplementary Figs. 8 and 9. Source data are provided as a source data file. 
a
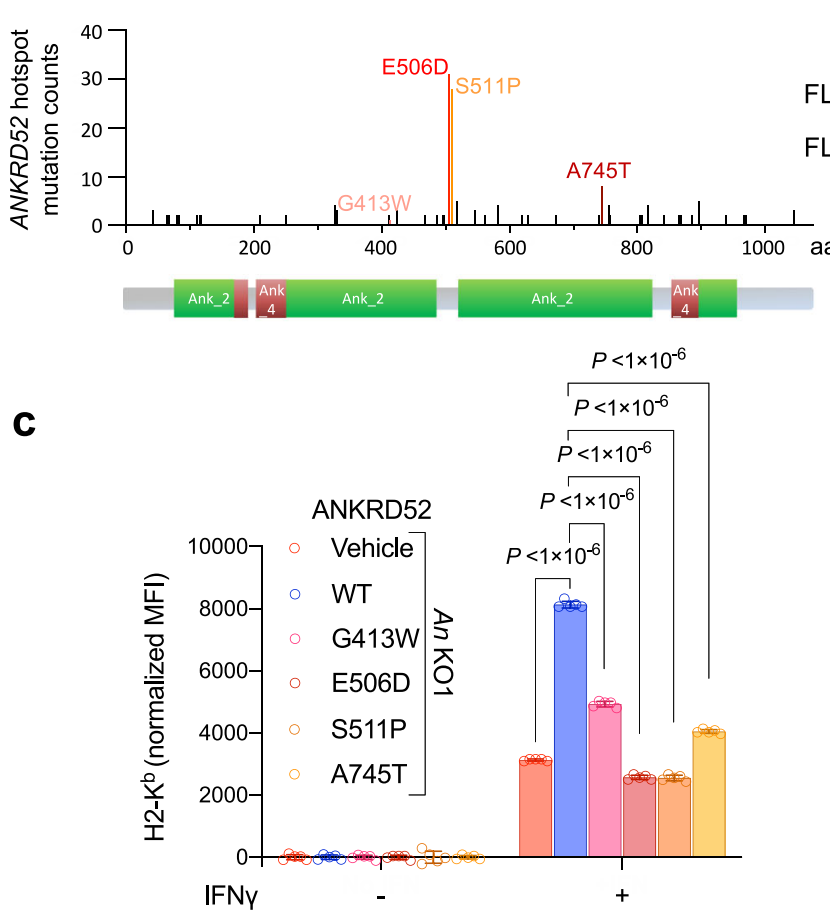

b

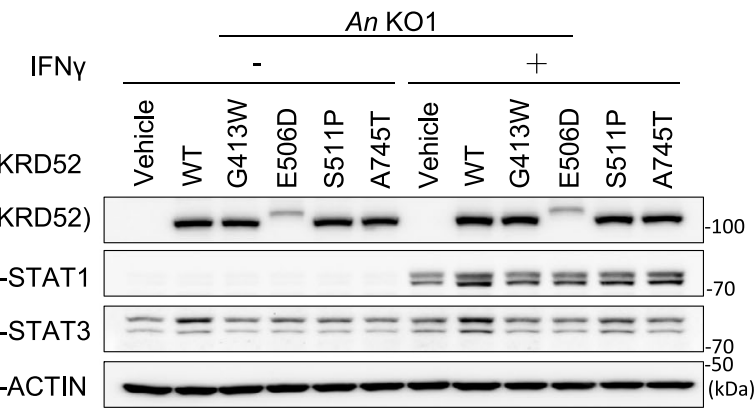

d

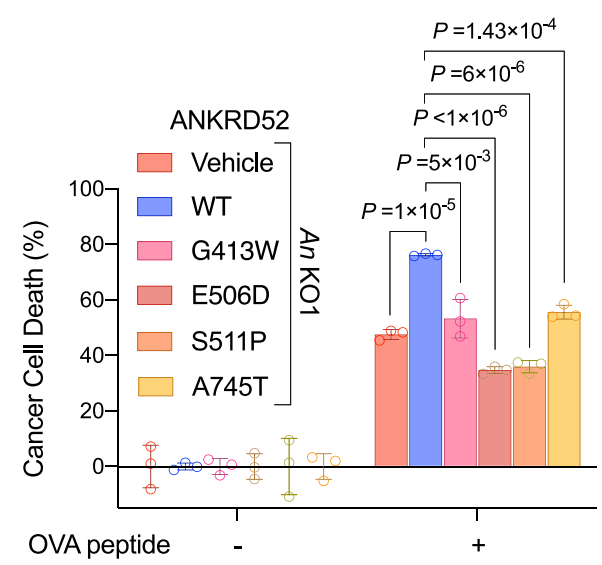

e

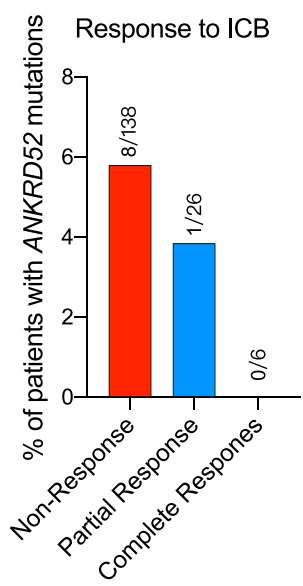

f

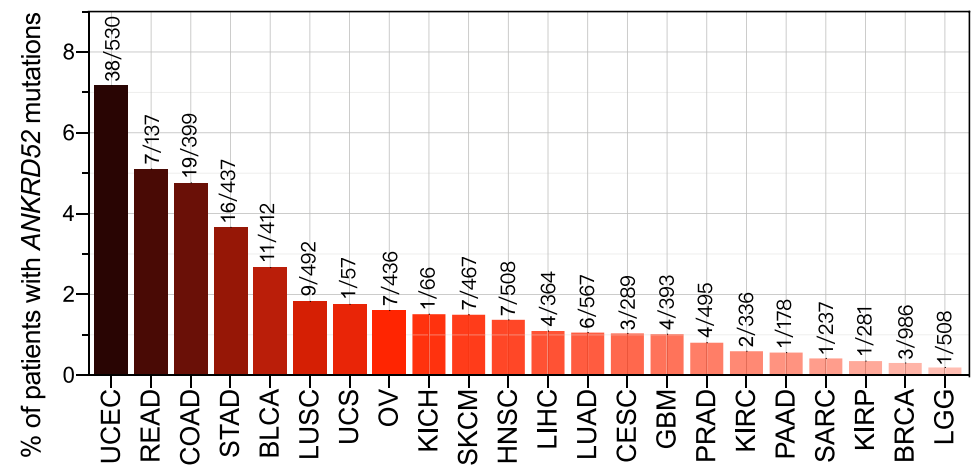

Fig. 5 Hotspot ANKRD52 mutations in patients impair IFN $\gamma$ and T cell response. a Localization of clinical hotspot ANKRD52 mutations from combined COSMIC and OncoWuXi database. b Protein abundance of p-STAT1 and p-STAT3 in Ankrd52-null MC38 cells expressing WT or indicated mutant ANKRD52 after IFN $\gamma$ treatment. Data are representative of two independent experiments. c Membrane MHC-I level in Ankrd52-null MC38 cells expressing WT or indicated mutant ANKRD52 after IFN $\gamma$ treatment. MFI of $\mathrm{H} 2-\mathrm{K}^{\mathrm{b}}$ was normalized by the responding group without IFN $\gamma$ treatment $(n=5$ per group per condition). Data are representative of two independent experiments and represented as mean \pm s.d., significance was determined using two-tailed unpaired Student's $t$-test. d Killing of OVA-treated Ankrd52-null MC38 cells expressing WT or mutant ANKRD52 by OT-I T cells ( $n=3$ per group per condition). Data are representative of two independent experiments and represented as mean \pm s.d., significance was determined using two-tailed unpaired Student's t-test. e Bar plot showing the percentage of patients with mutations in ANKRD52 across cancer patients receiving ICB therapies (anti-CTLA-4 or anti-PD-1) reported by Van Allen et al., Science 2015 and Riaz et al., Cell 2017. The non-response group is composed of SD and PD. f Bar plot showing the percentage of patients with mutations in ANKRD52 across multiple cancer types. Source data are provided as a source data file.

immunotherapies ${ }^{46,47}$ revealed a higher ANKRD52 mutation rate in tumor samples from non-responders than responders (Fig. 5e). Given a $4.8 \%$ prevalence of $A N K R D 52$ mutations (19/399) in colorectal adenocarcinoma and $1.5 \%(153 / 10182)$ in all cancer types (Fig. 5f), our studies suggest that these patients might not benefit as much from $\mathrm{T}$ cell-based immunotherapies.

Silencing of SOCS1 by ANKRD52-regulated miRNA targeting. ANKRD52, an Ankyrin repeat protein, forms a complex with the PPP6C phosphatase to dephosphorylate Argonaute 2 (AGO2) to promote miRNA-mediated gene silencing, a process counteracted by $\mathrm{CK} 1 \alpha$-induced phosphorylation of $\mathrm{AGO} 2^{21}$. Blocking CK1a rescued the impaired phosphorylation of STAT1 in IFN $\gamma$ stimulated ANKRD52-deficient cells (Fig. 6a; Supplementary Fig. 10a), implicating a potential role of the AGO2 phosphorylation cycle in regulating IFN $\gamma$ response.

We, therefore, hypothesized that certain miRNA-silenced targets were upregulated upon ANKRD52 deletion to suppress the IFN $\gamma$ response. Gene set enrichment analysis (GSEA) of MC38 transcriptome identified $19 \mathrm{IFN} \gamma$ signaling genes 
a

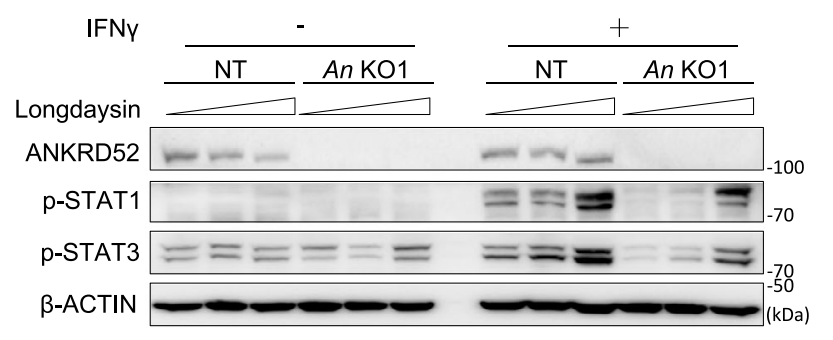

d

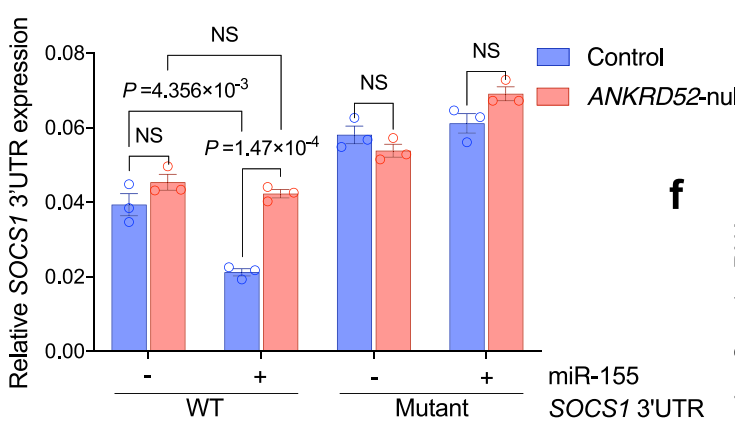

e
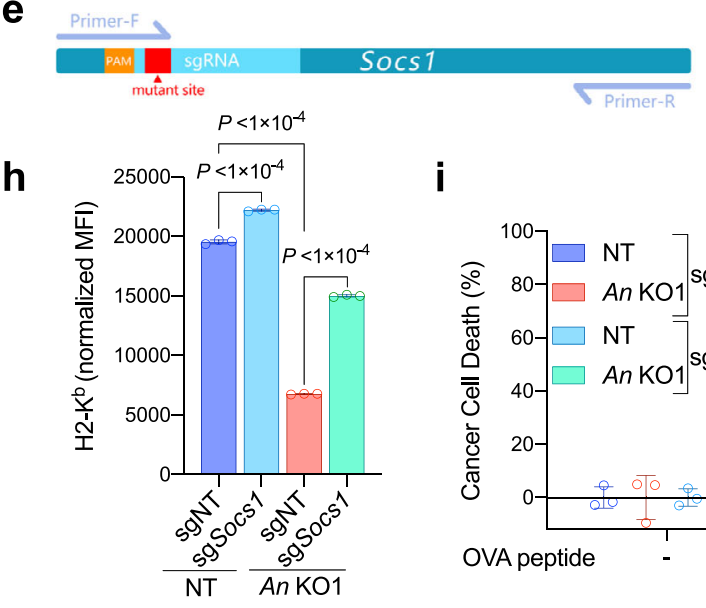

i b

b

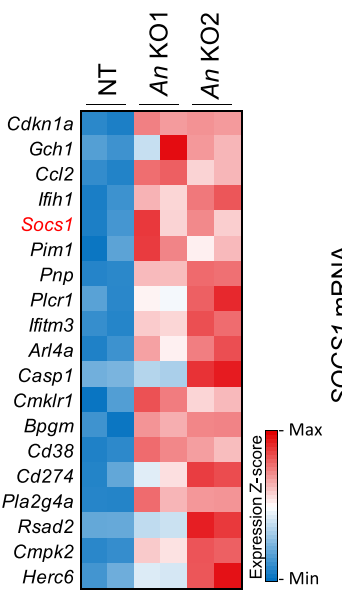

C

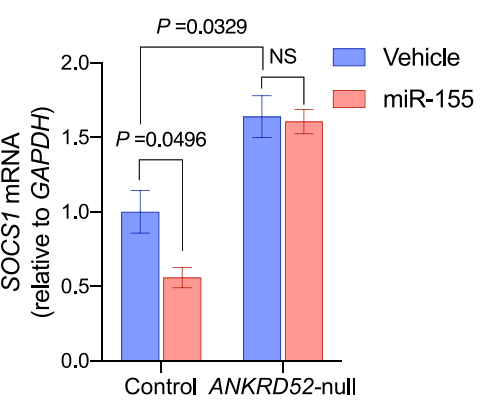

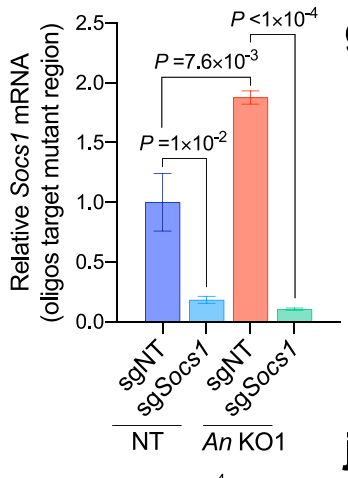

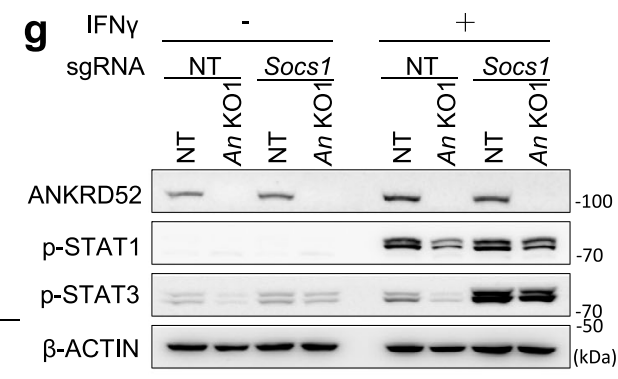

j

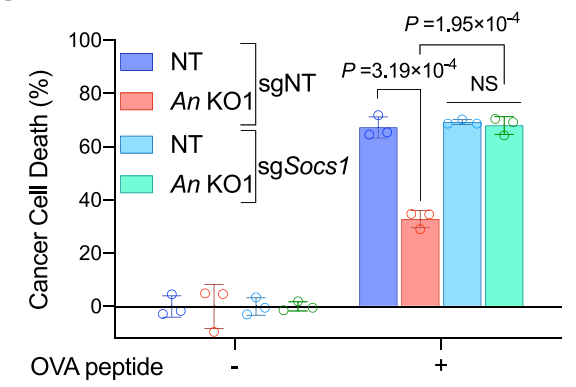

Fig. 6 ANKRD52 is required for miRNA-mediated silencing of SOCS1 to regulate $\mathbf{T}$ cell response. a p-STAT1 and p-STAT3 abundance in MC38 cells treated with IFN $\gamma$ and increasing Longdaysin $(0,50,100 \mu \mathrm{M}$ a CK1 $\alpha$ inhibitor). Data are representative of three independent experiments. $\mathbf{b}$ Heatmap showing commonly upregulated gene expression of IFN $\gamma$ signaling by RNA-seq analysis of Ankrd52-null MC38 cells ( $n=2$ replicates per group). c SOCS1 mRNA level in control and ANKRD52-null 293 T cells overexpressing miR-155 ( $n=3$ per group per condition). Data are representative of two independent experiments and represented as mean \pm s.e.m., significance was determined using two-tailed unpaired Student's $t$-test. d Activity of WT and mutant SOCS1 3'UTR (Rluc/Fluc) in a dual-luciferase reporter in 293 T cells overexpressing miR-155 ( $n=3$ per group per condition). Data are representative of three independent experiments and represented as mean \pm s.e.m., significance was determined using two-tailed unpaired Student's t-test. e Schematic overview of qPCR test targeting mutant region to check Socs 1 knockout efficiency. f Socs1 mRNA level tested by qPCR targeting mutant region in Ankrd52-null MC38 cells with inactivated SOCS1 ( $n=5$ per group). Data are represented as mean \pm s.e.m., significance was determined using two-tailed unpaired Student's $t$ test. $\mathbf{g}$ p-STAT1 and p-STAT3 abundance in Ankrd52-null MC38 cells with inactivated SOCS1 after IFN $\gamma$ treatment. Data are representative of three independent experiments. h Membrane MHC-1 expression in Ankrd52-null MC38 cells with inactivated SOCS1 after IFN $\gamma$ treatment. MFI of H2-Kb was normalized by the responding group without IFN $\gamma$ treatment ( $n=3$ per group per condition). Data are representative of two independent experiments and represented as mean \pm s.d., significance was determined using two-tailed unpaired Student's $t$-test. i Killing of OVA-treated Ankrd52-null MC38 cells with inactivated SOCS1 by OT-I T cells ( $n=3$ per group per condition). Data are representative of two independent experiments and represented as mean \pm s.d., significance was determined using two-tailed unpaired Student's $t$-test. $\mathbf{j}$ Volcano plot showing the Spearman's correlation and estimated significance of ANKRD52 with SOCS1 mRNA levels across all TCGA cancer types. Each dot represents a cancer type, blue dots indicate significant negative correlations $(P<0.05$, TIMER). See also Supplementary Figs. 10 and 11. Source data are provided as a source data file.

significantly upregulated in ANKRD52-deficient cells compared to control cells (Fig. 6b; Supplementary Fig. 10b; Supplementary Data 3), among which SOCS1 was also shown to be similarly controlled by ANKRD52 in human colorectal carcinoma HCT116 cells $^{21}$ (Supplementary Fig. 10c). SOCS1 suppresses JAK-STAT signaling and cancer immunotherapy ${ }^{11,45}$, and its mRNA levels were reciprocally controlled by ANKRD52 and CK1a (Supplementary Fig. $10 \mathrm{~d}$, e). Overexpression of miR-155, the major SOCS1-targeting miRNA ${ }^{48}$, in $293 \mathrm{~T}$ cells led to ANKRD52dependent repression of SOCS1 mRNA levels (Fig. 6c; 
Supplementary Fig. 10f). Using a luciferase reporter fused to the SOCS1 3'UTR harboring an intact or mutant miR-155 seed binding sequence ${ }^{48}$, we found that miR-155-mediated SOCS1 silencing required both ANKRD52 expression and correct miRNA targeting (Fig. 6d). CRISPR-mediated disruption of miR-155 in MC38 cells resulted in an increase of Socs1 mRNA level (Supplementary Fig. 11a), and a correspondent decrease of IFN $\gamma$-stimulated responses and $\mathrm{T}$ cell-mediated cytotoxicity (Supplementary Fig. 11b-g). Importantly, deletion of SOCS1 in Ankrd52-null MC38 cells (Fig. 6e, f) rescued the impaired STAT1 phosphorylation, the decreased membrane MHC-I level, and the dampened $\mathrm{T}$ cell-mediated cytotoxicity (Fig. 6g-i). Notably, TIMER analysis of the TCGA datasets ${ }^{49}$ revealed a strong negative correlation between transcript levels of ANKRD52 and SOCS1 in many human cancer types (Fig. 6j). Taken together, these results indicate that ANKRD52 regulates cancer immunity mainly through miR-155-mediated silencing of SOCS1.

Regulation of cancer immunity by miRNA machinery. Before targeted to complementary mRNA, mature miRNA is produced from precursor miRNA, which is cleaved from primary miRNA by the DROSHA-DGCR8 complex, exported from the nucleus to the cytoplasm by XPO5, and finally processed by DICER ${ }^{20}$. Analysis of 789 genome-wide CRISPR screen data (CRISPR Avana Public 20Q3, depmap.org) revealed a strong positive codependency between ANKRD52 and any of the core miRNA biogenesis and targeting machinery (Fig. 7a; Supplementary Fig. 12a). Like ANKRD52, transcript levels of these core miRNA processors negatively correlate with the SOCS1 level in a large number of human cancer types (Fig. 7b, c; Supplementary Fig. 12b-e). Consistently, CRISPR-mediated inactivation of Ago2, Dicer1, Xpo5, and Dgcr8 in MC38 cells increased Socs1 mRNA (Fig. $7 \mathrm{~d}$ ), and reduced downstream IFN $\gamma$-stimulated responses (STAT1 activation and antigen presentation), as well as mitigated $\mathrm{T}$ cell-mediated killing (Fig. 7e; Supplementary Fig. 12f-j, 13a-g, $14 \mathrm{a}-\mathrm{g})$. Moreover, MC38 tumors with inactivated AGO2 exhibited growth advantage in the immunocompetent host with increased $\mathrm{T}$ cell pressure induced by PD-1 blockade treatment (Fig. 7f; Supplementary Fig. 14h). Remarkably, analysis of TCGA datasets revealed that expression of these miRNA biogenesis (DICER1, XPO5, and DROSHA) and targeting (AGO2, PPP6C, and $A N K R D 52)$ machinery components all positively correlated with the intratumoral $\mathrm{T}$ cell abundance across almost all human cancer types, while SOCS1 expression did not exhibit such correlation (Fig. $7 \mathrm{~g}$ ).

Additionally, mutations in the miRNA machinery are found in 4-7\% CRC patient samples (Supplementary Fig. 15a). Some of these mutations were associated with worse survival of CRC patients exhibiting a higher degree of cytotoxic $\mathrm{T}$ lymphocyte infiltration (Supplementary Fig. 15b). In gene expression-based consensus molecular subtyping (CMS), a robust CRC classification system ${ }^{50}$, miRNA machinery mutations were enriched in CMS1 (Supplementary Fig. 15c), a subtype linked to strong immune activation, poor patient survival after relapse, and bleak prognosis in metastatic $\mathrm{CRC}^{50,51}$. Therefore, inactivation of the miRNA machinery not only protects tumor cells from $\mathrm{T}$ cellmediated cytotoxicity but also results in a pro-survival tumor microenvironment (Fig. 7h).

\section{Discussion}

Cancer cells accumulate a myriad of genetic and epigenetic alterations to enable escaping from host immune elimination ${ }^{33}$. It is a great challenge to single out those alterations driving immune evasion and to unravel how they empower cancer cells against hostile immunity ${ }^{7}$. Here, we combined immune selection and
CRISPR screen validation in vivo to identify spontaneous or rare mutations in cancer cells that endow resistance to $\mathrm{T}$ cell-mediated cytotoxicity. Many of the mutations affected single residue replacement (24/59; Fig. 1b) and may result in the gain of function in the corresponding proteins, which will not be well represented in our conventional CRISPR knockout screen. The interaction between these mutations and host immunity can be further explored by editing the cancer cell genome individually or in batch with the CRISPR base-editing tools ${ }^{52}$. Regardless, the same tumor profiling and CRISPR screening approach can be applied to different cancer cell lines and variable immune selection to untangle the cancer genetic heterogeneity, for identification of alterations that enable cancer cells to evade innate and adaptive immunity, and locating additional immunotherapeutic strategies from them. For example, we can profile mutations in syngeneic tumors from mice with or without depletion of NK cells or macrophages and interrogate genes critical for cancer cells to survive these innate immune cells. Alternatively, we can randomly mutagenize mouse cancer cell lines to increase the odds of mutant clone expansion upon selection from host immunity or immunotherapy during tumor growth or metastasis.

Loss of function mutation in Ankrd52 was enriched in cell linederived tumors grown specifically under T cell-mediated immune pressure (Fig. 1b). ANKRD52 forms a complex with PPP6C to counteract CK1 $\alpha$-mediated AGO2 phosphorylation and promote miRNA loading to target $\mathrm{mRNA}^{21}$. Like $A N K R D 52$, which is mutated in $4.8 \%$ colorectal adenocarcinoma and $1.5 \%$ of all cancer types (Fig. 5f), PPP6C harbors recurrent and potentially driver mutations, such as the $\mathrm{R} 264 \mathrm{C}$ mutation in $3 \%$ melanoma, which may disrupt PPP6C binding to ANKRD52 and thus the enzymatic activity ${ }^{28}$. Consistently, keratinocyte-specific deletion of $P p p 6 c$ promotes tumor formation in mice ${ }^{53}$. The other core miRNA pathway genes are also frequently mutated in cancer. DROSHA is frequently mutated in Wilms tumor samples with more than $70 \%$ mutations occurring at a metal-binding residue E1147 in the RNase III domain ${ }^{26,27}$. DICER1 is considered a haploinsufficient tumor suppressor, as loss of a single Dicer1 allele promotes tumorigenesis and reduces survival in mice ${ }^{29,54}$. Its mutations are frequently found in different cancer types and contribute to metastasis and poor survival in patients ${ }^{20,23-25,30}$. XPO5-inactivating mutations are detected in endometrial, colon, gastric, and breast tumors with microsatellite instability, causing impaired pre-miRNA export and are associated with increased cancer risk ${ }^{55,56}$.

Here, we show that the abundant mutations in these core miRNA machinery components may not only promote uncontrolled cancer cell-intrinsic proliferation but also enable them to resist $\mathrm{T}$ cell-mediated elimination and thus confer an additional survival advantage to cancer cells in a hostile immune environment. Inactivation of the machinery down-regulates $\mathrm{T}$ cell recruitment chemokines CXCL9 and CXCL10 (Fig. 4d-f), and remodels a more favorable microenvironment for tumor growth (Fig. 2h, i). In most human cancers, expression of the core machinery components exhibits very strong positive correlations with tumor-intrinsic T cell infiltrations (Fig. 7g). Notably, CXCL9 expression is regarded as a strong predictor of ICB response in a meta-analysis of over 1000 ICB-treated patients across seven tumor types ${ }^{57}$. Our results suggest that a sizeable patient subpopulation with defective miRNA activity may not respond well to $\mathrm{T}$ cell-based immunotherapies.

Despite the recent remarkable clinical success of PD-1/PD-L1 ICB, most cancer patients fail to respond or develop treatment resistance, frequently due to defective antigen presentation and IFN $\gamma$ signaling $1,4,13,14$. We demonstrate that the core miRNA biogenesis and targeting machinery are essential for the IFN $\gamma$ activated JAK-STAT signaling and antigen presentation in cancer 
a
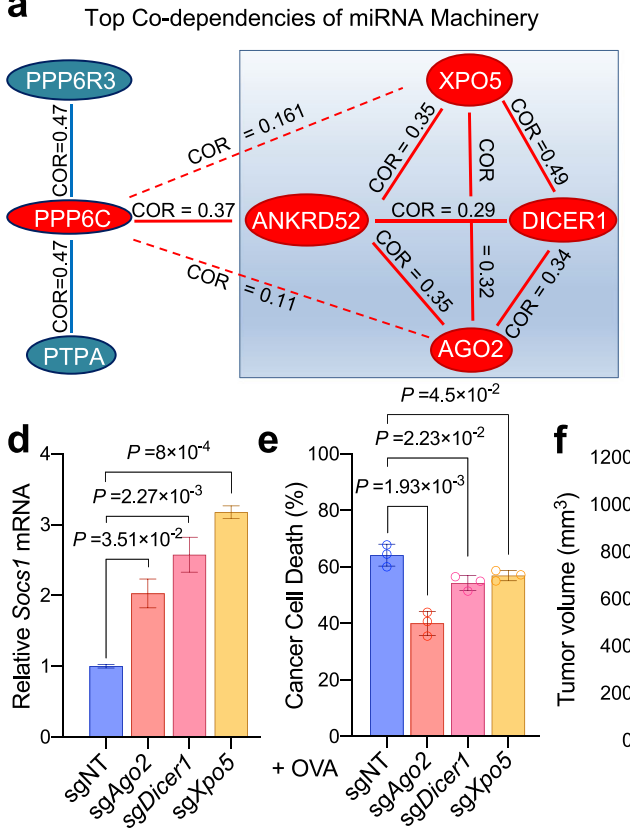

b

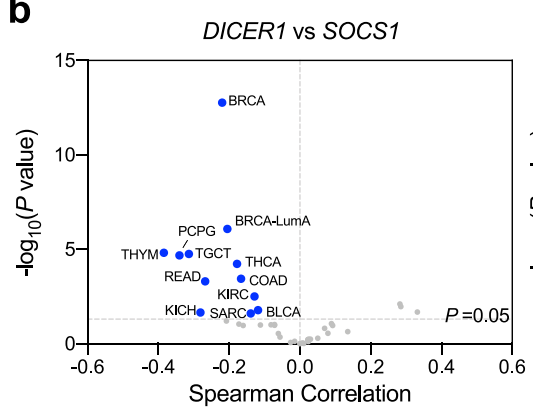

C

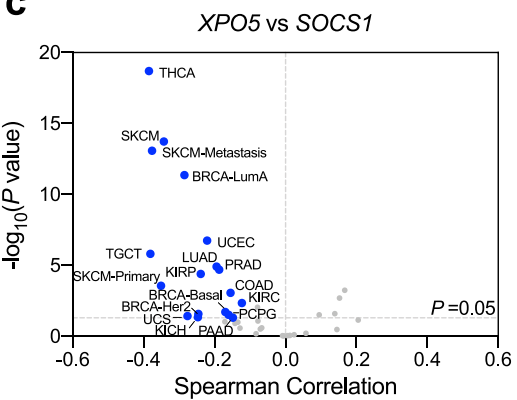

h
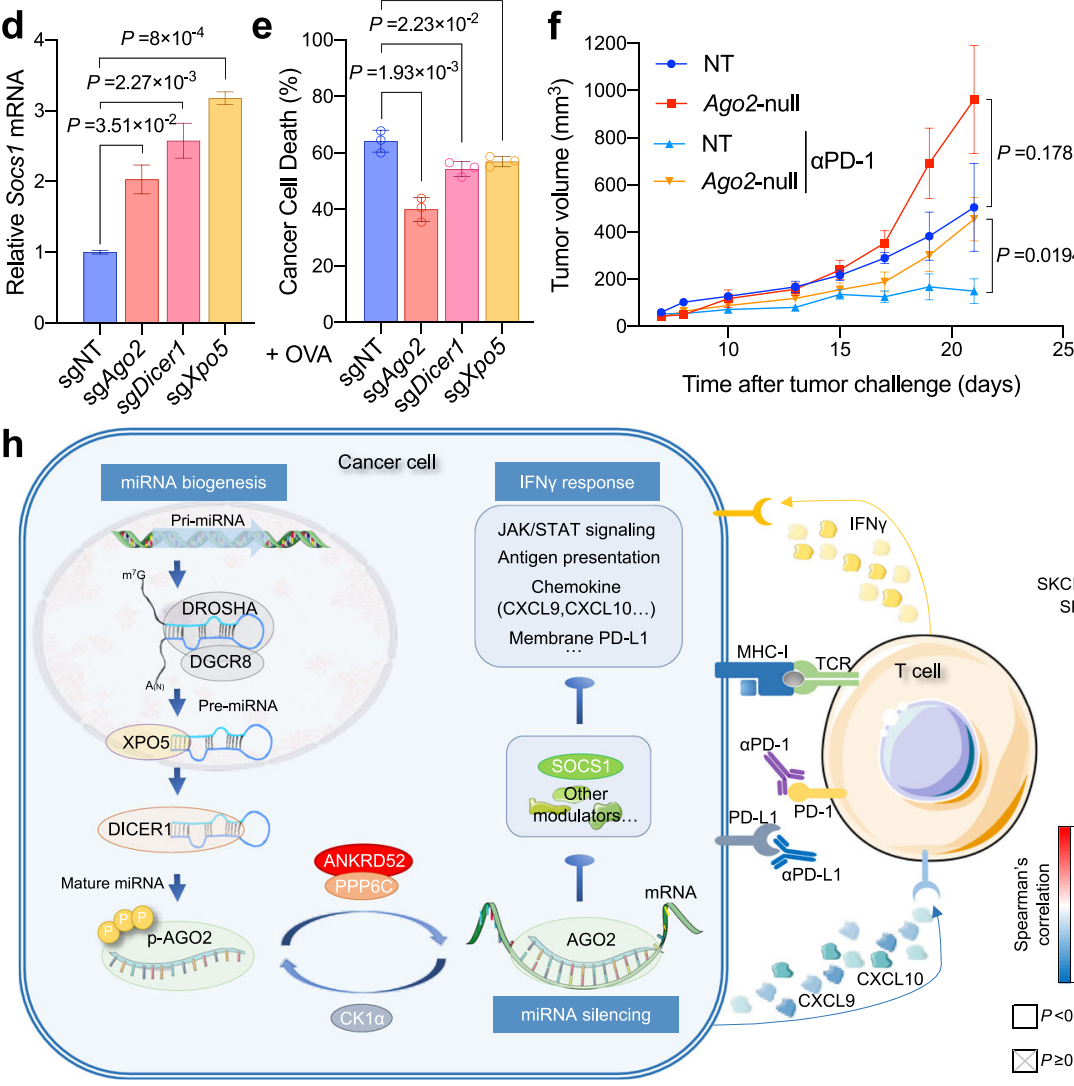

g

$\mathrm{CD} 4^{+} \mathrm{T}$ cell infiltration $\mathrm{CD} 8^{+} \mathrm{T}$ cell infiltration

$$
\text { Time after tumor challenge (days) }
$$

$25_{\text {BRCA-Basal }(n=191)}$

BRCA-Her2 $(n=82)$ BRCA-LumA $(n=568)$

CESC $(n=306)$ CHOL $(n=36)$ $\operatorname{COAD}(n=458)$ COAD $(n=458)$ TGCT $(n=150)$
THCA $(n=509)$ SKCM $(n=471)$ SKCM-Metastasis $(n=368)$ SKCM-Primary $(n=103)$ PAAD $(n=179)$
$\operatorname{READ}(n=166)$ READ $(n=166)$
SARC $(n=260)$ STAD $(n=415)$ $\operatorname{ACC}(n=79)$ $\operatorname{BLCA}(n=408)$ DLBC $(n=48)$ $\operatorname{LIHC}(n=371)$
$\operatorname{UCS}(n=57)$ UCS ( $n=57)$ GBM $(n=153)$
HNSC $(n=522)$
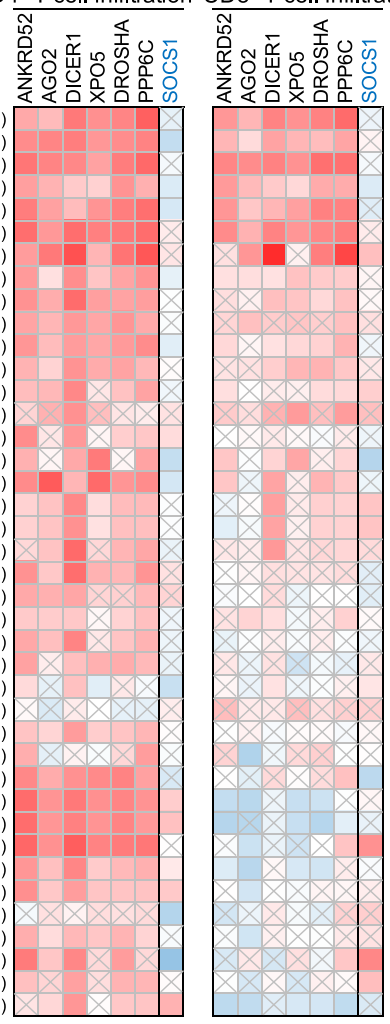

Fig. 7 Inactivation of miRNA machinery promotes cancer-intrinsic escape from T cell elimination. a Diagram showing the Spearman's correlation of top co-dependent proteins with ANKRD52 or PPP6C in CRISPR (Avana) Public 20Q3 database. Solid lines depict significant positive correlations (Correlation $>0.25, P<0.001$ ) and dashed lines depict weak correlation (Correlation $>0.1, P<0.01$ ). $\mathbf{b}$, c Volcano plot showing the Spearman's correlation and estimated significance of DICER1 (b) or XPO5 (c) with SOCS1 mRNA levels from RNA-seq data across all TCGA cancer types. Each dot represents a cancer type in TCGA; blue dots indicate significant negative correlations $(P<0.05$, TIMER). d SOCS1 mRNA level in MC38 cells with targeted sgRNAs ( $n=3$ per group). Data are representative of three independent experiments and represented as mean \pm s.e.m., significance was determined using twotailed unpaired Student's t-test. e Killing of OVA-treated MC38 cells with targeted sgRNAs by OT-I T cells ( $n=3$ per group per condition). Data are representative of two independent experiments and represented as mean \pm s.d., significance was determined using two-tailed unpaired Student's $t$-test. f Tumor growth curves of Ago2-null or control MC38 tumors in WT mice treated with PD-1 antibody or not $(n=5$ for NT tumor, $n=6$ for NT with anti-PD1 and $n=7$ for Ago2-null with or without anti-PD-1). Data are represented as mean \pm s.e.m., significance was determined using two-tailed unpaired Student's t-test. $\mathbf{g}$ Heatmap showing the Spearman's correlation of ANKRD52, AGO2, DICER1, XPO5, DROSHA, PPP6C, or SOCS1 mRNA levels with CD4+ and $\mathrm{CD}^{+} \mathrm{T}$ cell abundance in tumors across all TCGA cancer types. $\mathbf{h}$ Model of miRNA machinery in regulation of cancer-intrinsic evasion from T cell attack. See also Supplementary Figs. 12-15. Source data are provided as a source data file.

cells, largely by controlling miR-155-targeted silencing of SOCS1 (Fig. 7h). Unlike our observation in CRC cells, Dicer inactivation was reported to result in increased IFN signaling via Let-7 miRNA in macrophages and embryonic stem cells ${ }^{58,59}$, both primary cells. This discrepancy may be due to the heterogeneous mutations in cancer cells that dictate distinct IFN response upon Dicer inactivation. IFN $\gamma$ signaling is regulated by multiple positive and negative feedbacks, thus playing different or even opposing roles in cancer cells and immune cells ${ }^{41,60,61}$.
Interestingly, depletion of SOCS1 was also found to sensitize cancer cells to PD-1 blockade in the previous in vivo CRISPR library screen ${ }^{11}$. Although expression levels of individual miRNA machinery components are inversely correlated with SOCS1 in many human cancer types (Fig. 6j; Fig. 7b, c), inactivation of the core miRNA pathway should block silencing of many more genes in addition to SOCS1 for immune response, which still need to be further elucidated. Additionally, we showed that chemical inhibition of the CK1a kinase activity can counteract the decreased 
IFN $\gamma$ response (Fig. 6a). CK1a could also be degraded by lenalidomide ${ }^{62}$, a clinically proved anti-myeloma drug reported to enhance $\mathrm{T}$ cell-mediated anti-tumor activity and ICB response ${ }^{63}$. Our data suggest that targeting the miRNA machinery in combination with immunotherapies may provide a cancer therapeutic strategy.

\section{Methods}

Animals. The protocol and any amendment(s) or procedures involving the care and use of animals in this study were in accordance with Shanghai Institutional Animal Care and Use Committee (IACUC) guidelines and approved by IACUC of ShanghaiTech University or WuXi AppTec. Animal experiments were conducted at the National Facility for Protein Science or OIU of WuXi AppTec. C57BL/6 J and Foxn $1^{\text {nu }}$ (nude) mice (female, aged at 6-8 weeks and weighing approximately 18-22 g) were used for tumor implantation experiments and purchased from Shanghai SLAC Laboratory Animal. Mice were housed at approximately $20-26^{\circ} \mathrm{C}$, $40-70 \%$ humidity on a 12 -hr light/dark cycle in a special pathogen-free environment and in individual ventilation cages (four mice per cage). Tumor burden did not exceed $10 \%$ of the animal's body weight for each mouse.

Cell lines and cell culture. All cell lines used in this study were tested as mycoplasma-negative using the Universal Mycoplasma Detection Kit (ATCC, 301012 K). 293FT (R70007) and 293 T/17 (CRL-11268) cell lines were originally purchased from Thermo Fisher and ATCC, respectively, and cultured according to the manufacturers' manual specifically. MC38 cell line was provided by WuXi AppTec and cultured using DMEM (Gibco) with 10\% fetal bovine serum (FBS), respectively.

MC38-OVA cell lines were constructed by introducing cDNA of OVA peptide into the plenti6.3 vector (Thermo Fisher, K533000). The cDNA sequences for OVA peptide (p.257-264, SIINFEKL) were synthesized at GENEWIZ. Blasticidin (Gibco, A1113903) selection was used for lentivirus transduced cells $(\mathrm{MOI} \approx 0.3$ ) to generate OVA stably expressing cells.

OT-I T cells were isolated from the spleen and lymph nodes of OT-I transgenic mouse (a gift from Bing Sun's Lab at SIBCB) by EasySep ${ }^{\text {TM }}$ Mouse CD8 ${ }^{+}$T Cell Isolation Kit (STEMCELL, 19753). Fresh isolated CD8 ${ }^{+} \mathrm{T}$ cells were cultured in a complete T cell medium (RPMI-1640 with 10\%FBS, 20 mM HEPES, $1 \mathrm{mM}$ sodium pyruvate, $0.05 \mathrm{mM}$ 2-mercaptoethanol, $2 \mathrm{mM}$ L-glutamine, $50 \mathrm{U} / \mathrm{ml}$ streptomycin and penicillin), and treated with $2 \mathrm{ng} / \mathrm{mL}$ recombinant mouse IL-2 (Novoprotein, CK24).

In vivo tumorigenesis under different immune selection. Murine tumor syngeneic model under different immune selective pressure was built using nude or C57BL/6 J mice with or without different antibody or IgG treatment. Each mouse was inoculated subcutaneously at the right flank with MC38 tumor cells $\left(1 \times 10^{6}\right.$ / mouse) in $0.1 \mathrm{~mL}$ of PBS for tumor development. Tumor measurement was taken manually by collecting the longest dimension (length, $L$ ) and the longest perpendicular dimension (width, $W$ ). Tumor volume was estimated with the formula: $\left(L \times W^{2}\right) / 2$. When the average tumor volume reached approximately $40-60 \mathrm{~mm}^{3}$, the animals were performed with stratified randomization empirically based upon their tumor volumes and treatment was started. The test article administration for groups in all experiments of this research are shown in Table 1.

All the antibodies for mice injection were purchased from Bio X Cell and listed as follows: anti-PD-1 (BE0146), anti-PD-L1 (BE0101), anti-CD4 (BE0119), antiCD8 (BE0117), IgG2a (BE0089), and IgG2b (BE0090). The study was terminated for tumor collection before the mean tumor volume of any immunodeficient group reached a value of $2000 \mathrm{~mm}^{3}$.

RNA-Seq analysis. For tumors, collected tumors were cut large into samples with size $\leq 0.5 \mathrm{~cm}$ in dimension and then the fresh tissues were placed in 5-10 volumes of RNAlater ${ }^{\circledR}$ Solution (Thermo Fisher, AM7020). For cultured cells, Ankrd52-null or control MC38 cells were seeded onto 6-well plates at $5 \times 10^{5}$ cells/well in duplicate and challenged with $10 \mathrm{ng} / \mathrm{ml} \mathrm{IFN} \gamma$ (CST, 39127) for $24 \mathrm{~h}$, with cytokinefree medium serving as control. RNA isolation, library construction, and sequencing were performed by Mingma Technologies. RNeasy MinElute Cleanup Kit (QIAGEN, 74204) was used for RNA extraction, followed by generating mRNA- focused sequencing libraries from total RNA using Illumina TruSeq RNA Sample Preparation Kit v2. Paired-end 150 bp sequencing was done on illumina Hiseq X10 ( $40 \mathrm{M}$ reads for each sample). FastQC (0.11.2) was used to generate QC report and the adapters of each sample were trimmed, respectively.

To measure gene expression level, fastq file of each sample was mapped to the reference genome to get reads count table as input for differential expression analysis. The clean reads were mapped to the mouse (Mus musculus) genome (mm10) using STAR ${ }^{64}$ (2.4.2a) and annotated with a transcriptome database (gene code vM13). Gene abundance estimation by read counts was conducted with the software RSEM ${ }^{65}$ (1.2.29). Normalized tags per million (TPM) were calculated on the number of clean reads mapped to a specific region of the genome using the relative log expression (RLE) method in edge $\mathrm{R}^{66}$ (3.16.5). Differentially expressed genes (DEG) refer to compared gene expression levels between two samples or two groups. DEG were defined by using the criterion that fold-change $>1.5$ and adjust $P<0.05$ (adjust $P$-value). Pathway enrichment analysis (GO and KEGG) on DEGs was performed on differentially expressed genes using GOstats ${ }^{67}(2.40 .0)$ in R, with the threshold that $P<0.01$. Gene set enrichment analysis for molecular signature ${ }^{68}$ was carried out using GSEA ${ }^{69}$ software with threshold that $P<0.01$. Tumorinfiltrating lymphocytes were analyzed by mMCP counter ${ }^{70}$.

Identification of mutations from profiled cancer heterogeneity. To detect mutations (SNP and InDel) in RNA-seq datasets from tumors under different immune selection, we used the aligner STAR (2.5.2b) to map the reads to the genome (GRCm38 - mm10) and used MarkDuplicates in picard-tools-1.94 (http:// broadinstitute.github.io/picard) to dedup the bam file ${ }^{64}$. And then, we applied a joint call to detect SNP and Indels across all RNA-seq datasets by following the GATK $^{36}$ (3.7.0) best-practice guidelines (https://gatk.broadinstitute.org/hc/en-us/ sections/360007226651-Best-Practices-Workflows). In the variant filtering step, we used aspecific hard filter to get the filtered VCF file. Then, we used ANNOVAR ${ }^{71}$ to annotate the mutation sites. Finally, we only kept the mutations in exonic and splicing regions for further study. Mutation allele frequency was counted with the formula: depth of the alteration/total depth of the allele at a particular locus.

To profile the mutations, we compared the mutations from tumors under different immune selection and enriched hotspot mutations as rules showed in Table 2.

mMCE pooled sgRNA library construction. The oligonucleotide pool targeting 53 candidate genes from profiled heterogeneity and controls (ten individual sgRNAs per gene) was customized at CustomArray, Inc. Then, the oligo pool was annealed and subcloned into lentiCRISPRv2 (Addgene, 52961) backbone using the Gibson Assembly Kit (NEB) and electroporation according to the protocol from Zhang's lab $^{72}$. Library representation was maintained for at least $1000 \times$ coverage at each step of the process. To qualify the sgRNA representation, the constructed library was amplified by two-step PCRs using Phusion ${ }^{\circledR}$ Hot Start Flex DNA Polymerase (NEB, M0535L), in which the second step of PCR was conducted using the PCR amplicon from the first PCR as template. The primers for the first step of PCR are 5'-AATGGACTATCATATGCTTACCGTAACTTGAAAGTATTTCG-3' (Forward) and $5^{\prime}$-TAGGCACCGGATCAATTGCCGAC-3' (Reverse). The primers for the step of PCR are $5^{\prime}$-(1-9 bp of variable length sequence)TCTTGTGGAAAGGACGAAACACCG-3' (Forward) and $5^{\prime}$-(1-9 bp of variable length sequence) TGTGGGCGATGTGCGCTCT-3' (Reverse). The amplicon was sent for nextgeneration sequencing (NGS) at Mingma Technologies using illumina Hiseq X10 (3.3 $\mathrm{M}$ reads, $1 \mathrm{G}$ data) to qualify sgRNA library distribution before screening ${ }^{72}$.

Lentivirus production. To produce lentivirus, $3 \times 10^{7} 293 \mathrm{FT}$ cells were collected for reverse-transfection in each T150 flask. For each T150 flask, $15 \mu \mathrm{g}$ library or other sgRNA plasmid, $11.25 \mu \mathrm{g}$ psPAX2 (Addgene, 12260), and $7.5 \mu \mathrm{g}$ pCMVVSV-G (Addgene, 8454) diluted in $2 \mathrm{ml}$ Opti-MEM (Gibco, 11058021) were mixed with $100 \mu \mathrm{L}$ Lipofectamine 2000 (Invitrogen, 11668019) diluted in $2 \mathrm{ml}$ Opti-MEM. The mixture was added into 293FT cells and the medium was refreshed $12 \mathrm{~h}$ post transfection. Virus supernatant was collected $48 \mathrm{~h}$ post refreshing medium, centrifugated at $1,800 \mathrm{~g}$ at $4{ }^{\circ} \mathrm{C}$ for $10 \mathrm{~min}$ to pellet the cell debris and filtrated with a $0.45 \mu \mathrm{m}$ low protein binding membrane (Millipore, SE1M003M00). The lentivirus was concentrated by ultra-centrifugation at $25,000 \mathrm{rpm}$ at $4{ }^{\circ} \mathrm{C}$ for $2 \mathrm{~h}$, and then resuspended and aliquoted to store at $-80^{\circ} \mathrm{C}$.

Table 1 Conditions for in vivo tumorigenesis.

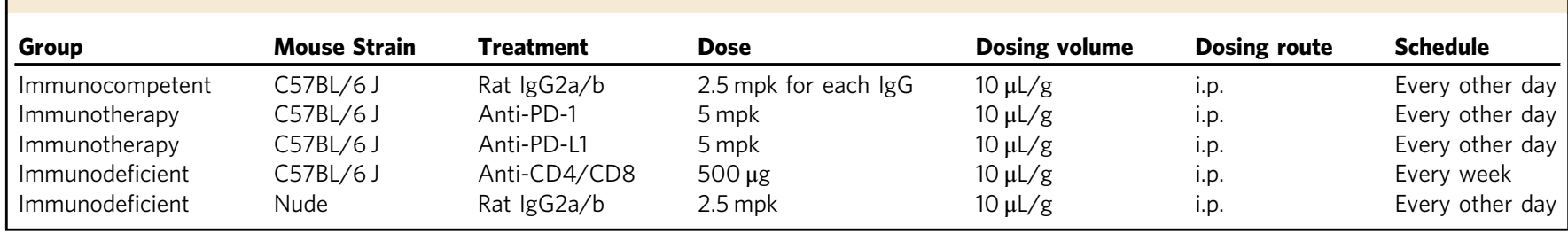


Table 2 Filter criteria for hotspot mutation profiling.

Category/group

Category 1:PD-1 dependent immune suppressor candidates

Category 2:Non-PD-1 immune suppressor candidates

\section{In vitro}

\section{Negative Negative}

Negative Negative
Immunodeficient Immunocompetent

C57BL/6 J

Positive in at least two of these tumors

Positive in at least two of these tumors
Immunotherapy

$\alpha$ PD-L1 $\alpha$ PD-1

Negative

Positive in

any tumor

(Positive, $\mathrm{AF} \geq 0.1$; Negative, $\mathrm{AF}<0.1$ )

Lentivirus infection. To test the lentivirus titer, MC38 or MC38-OVA cells were seeded at $5 \times 10^{5}$ /well in 6 -well plates and infected by lentivirus at gradient dilution with polybrene $(7.5 \mu \mathrm{g} / \mathrm{mL})$ added $24 \mathrm{~h}$ later. $24 \mathrm{~h}$ post infection, cells were collected and re-seeded $\left(1 \times 10^{3}\right.$ cells/well in 96-well plates) with 6 replicates for each dose. The next day, according to the vector backbones, puromycin (Gibco, A1113803), hygromycin B (Invitrogen, 10687010), or G418 (Gibco, 10131027) was added to 3 of 6 replicated wells at $2.5 \mu \mathrm{g} / \mathrm{ml}, 100 \mu \mathrm{g} / \mathrm{ml}$ or $500 \mu \mathrm{g} / \mathrm{ml}$, respectively, for 7 days and then cell viability was detected by CellTiter-Glo Kit (Promega, G7570).

According to the virus titer, concentrated lentivirus infection at a low $\mathrm{MO}$ ( $\sim .3)$ was conducted for MC38 or MC38-OVA cells with polybrene added. The transduced cells were treated with puromycin, hygromycin B or G418 starting from 2 days post infection and kept selection for 7 days. Selected cells were maintained with a low concentration of puromycin $(0.5 \mu \mathrm{g} / \mathrm{ml})$, hygromycin B $(50 \mu \mathrm{g} / \mathrm{ml})$ or G418 $(200 \mu \mathrm{g} / \mathrm{ml})$. Antibiotics were removed 2 days before tests.

In vivo CRISPR screen. mMCE sgRNA library was transduced into MC38 cells for tumorigenesis in nude or C57BL/6 J mice with different treatments to validate profiled tumor heterogeneity. Together with inoculation, $2 \times 10^{6}$ cultured MC38 cells with mMCE library were collected as in vitro baseline. Tumors were randomly divided into the following groups, nude $(n=8)$ mice or WT mice with IgG $(n=8)$, anti-PD-1 $(n=10)$, anti-PD-L1 $(n=10)$, or anti-CD4/8 $(n=7)$, for treatment as described. Before treatment, five additional WT tumors were collected as in vivo baseline. And, 9 days after treatment, tumors (volume $=50 \sim 1500 \mathrm{~mm}^{3}$ ) were collected and grinded into homogeneous powders in liquid nitrogen. Genomic DNA was extracted by Blood \& Cell Culture DNA Midi Kit (QIAGEN,13343) from the powders. PCR amplification was conducted using $1 \mu \mathrm{g}$ of the genomic DNA as a template following the protocol for sgRNA library plasmid NGS sampling ${ }^{72}$. For each sample, 6 of $50 \mu \mathrm{l}$-reactions were performed for the first step of PCR and 2 of $50 \mu \mathrm{l}$-reactions were performed for the second step of PCR. Paired-end $150 \mathrm{bp}$ NGS was performed on the amplicons from the second PCR using an Illumina Hiseq $\mathrm{X} 10$ to determine sgRNA abundance (3.3 $\mathrm{M}$ reads, $1 \mathrm{G}$ data per sample).

T cell co-culture screen and assay. OT-I T cells were isolated from spleen and lymph nodes of OT-I transgenic mouse and stimulated with $2 \mathrm{ng} / \mathrm{mL}$ recombinant mouse IL-2 (Novoprotein, CK24), $5 \mathrm{ng} / \mathrm{mL}$ mouse IL-7 (Peperotech, 217-17) and $100 \mathrm{ng} / \mathrm{mL}$ mouse IL-15 (Peperotech, 210-15) for 2 days. Then, T cells were cocultured with tumor cells stimulated with $1 \mu \mathrm{M}$ SIINFEKL peptide (OVA p257264 , GL Biochem, 53,698) for $2 \mathrm{~h}$ or tumor cells stably expressing OVA peptide in the complete $\mathrm{T}$ cell medium with $2 \mathrm{ng} / \mathrm{mL}$ mouse IL-2 added.

For T cell co-culture screen, MC38-OVA cells transduced with mMCE sgRNA library post puromycin selection were seeded at $2 \times 10^{6}$ cells (library coverage $>2000 \times$ ) in each T750 flask (total 12 flasks). The next day, $2 \times 10^{6}$ effector $\mathrm{T}$ cells were planted in each flask with $2 \mathrm{ng} / \mathrm{mL}$ mouse IL- 2 added. $4 \times 10^{6} \mathrm{MC} 38$ OVA cells (library coverage $>4000 \times$ ) were collected as the baseline. With no-T cells as the negative control, tumor cells were collected at day $1\left(10 \times 10^{6}\right.$ cells $)$, day $2\left(4 \times 10^{6}\right.$ cells $)$ and day $2.5\left(1-2 \times 10^{6}\right.$ cells, library coverage $\left.>1000 \times\right)$ post coculture for NGS as described in the in vivo screen ( $n=3$ for each group per day).

For T cell co-culture assay, 200 genome-edited OVA expressing or treated $(1 \mu \mathrm{M}$ for $2 \mathrm{~h})$ tumor cells were plated onto 96 -well plates and co-cultured with extracted T cells for 2 days. T cells were analyzed by flow cytometry. Tumor cells post co-culture were analyzed by CellTiter-Glo Kit (Promega, G7570) for cell viability.

Data process and analysis for CRISPR screen. MAGeCKFlute was used to generate read counts for each sgRNA based on fastq files from paired-end NGS of PCR amplicons ${ }^{73}$. In order to ensure reliable ranking in depletion analysis, we tried to filter sgRNAs with low reads which could be a consequence of insufficient sampling of NGS. The mapped reads from all samples are over 30\% (from 30.65\% to $34.43 \%$ for in vivo screen, and from $33.88 \%$ to $34.43 \%$ for $\mathrm{T}$ cell co-culture screen, respectively) for $\mathrm{PE}$ reads, which means over $60 \%$ for one side covering the sgRNA region in the PCR product. In the filtering step for sgRNAs with low counts, we found no sgRNA with zero count. Both mapping rates and zero-count percentage demonstrate the high quality of each sample's NGS data for both the in vivo and in vitro screen. Then, the read counts for each sgRNA were normalized according to the formula as follows: Normalized reads per sgRNA $=$
Reads per $s g R A$ $\frac{\text { Reads per sgRNA }}{\text { Total count of mapped reads }} \times($ Max mapped reads count among all samples $)+1$. Tumorintrinsic immune evasion gene hits were determined based on both integrated beta score ranking (generated by MAGeCK-RRA ${ }^{73}$ ) and $\log _{2}$ fold-change (generated by edgeR ${ }^{74}$ ) with relevant $P$ value $(<0.05)$ in the comparisons of immunocompetent vs immunodeficient from in vivo screen and OT-I T cell $v s$ No $\mathrm{T}$ cell from $\mathrm{T}$ cell co-culture screen.

Construction of genome-engineered cell lines. MC38 and MC38-OVA cells with sgRNA targeting Ankrd52, Ppp6c, Ago2, Dicer1, Xpo5, or non-targeting control were generated using lentiCRISPRv2 (Addgene, 52961) vectors harboring annealed relevant sgRNA oligonucleotides. The sgRNA vectors were packaged into lentivirus and infected the mentioned cell lines $(\mathrm{MOI} \approx 0.3)$. Cells with sgRNAs were selected with $2.5 \mu \mathrm{g} / \mathrm{ml}$ puromycin (Gibco, A1113803) for $72 \mathrm{~h}$ and maintained with a low concentration of puromycin $(0.5 \mu \mathrm{g} / \mathrm{ml})$. Knockout efficiency was evaluated by WB analysis. Base on it, Ankrd52-null or control MC38 cells were generated from MC38 cells with sgRNA targeting Ankrd52 or non-targeting control by limiting dilution and single-cell clonal expansion. Confirmation of gene knockout was performed using QIAamp DNA Blood Mini kit (Qiagen), and sgRNA target regions were amplified by PCR followed by Sanger sequencing for frame-shift mutations. CRISPR-mediated Ankrd52 knockouts were also validated by RNA-Seq reads at the sgRNA targeting sites. Meanwhile, ANKRD52-null $293 \mathrm{~T}$ cells were generated using px459 (Addgene, 62988) vectors introduced with sgRNA targeting ANKRD52. $293 \mathrm{~T}$ cells were transfected with the sgRNA vector by Lipofectamine 3000 reagent (Invitrogen) and selected with puromycin $(1 \mu \mathrm{g} / \mathrm{ml})$ for $72 \mathrm{~h}$. Selected cells were subjected to limiting dilution for single-cell clonal expansion and confirmation of knockout. Moreover, Ankrd52-null or control MC38 cells with sgRNA targeting Socs 1 or non-targeting control were generated using pLVX-hyg-sgRNA (TaKaRa, 632632) vectors with relevant sgRNA by hygromycin B (Invitrogen, 10687010 ) selection following viral infection $(\mathrm{MOI} \approx 0.3)$. All the sgRNA sequences are listed in Supplementary Data 4

Ankrd52 mutant or recombinant cells were generated by stably overexpressing WT or clinic hotspot mutant Ankrd52 in Ankrd52-null MC38 cells. The WT cDNA sequences for Ankrd52 were designed with sgRNA-targeting-defective synonymous mutations and a FLAG-tag added at N-terminal, and synthesized at GENEWIZ. The cDNA was subcloned into pLVX-IRES-Neo vector (Clontech, 632181) and introduced with clinic hotspot mutations using Phusion Site-Directed Mutagenesis Kit (Thermo Fish, F541). Ankrd52-null MC38 cells were introduced with the cDNA vectors, respectively, by lentivirus $(\mathrm{MOI} \approx 0.3$ ) and selected by G418 (Gibco, 10131027)

In vivo competition assay. MC38 cells were transduced with four individual sgRNAs targeting Ankrd52 and three individual sgRNAs targeting non-targeting control. These sgRNA cells were mixed in equal proportions and inoculated into nude or C57BL/6 J mice. Mice were euthanized 16 days after tumor inoculation for tumor collection when the mean tumor volume in nude mice reached $1000 \mathrm{~mm}^{3}$ Tumor were grinded into homogeneous powders in liquid nitrogen for genomic DNA extraction and NGS. sgRNA distribution was analyzed by edgeR for $\log _{2}$ foldchange $\mathrm{e}^{74}$

Analysis of tumor-infiltrating immune cell populations. Ankrd52-null and control tumors were harvested at $\sim 1000 \mathrm{~mm}^{3}$ and cut up on ice for the following incubation in collagenase D ( $1 \mathrm{mg} / \mathrm{ml}$, Roche), DNase I (50 $\mu \mathrm{g} / \mathrm{ml}$, Sigma-Aldrich) and Hyaluronidase $(100 \mu \mathrm{g} / \mathrm{mL}$, Sigma-Aldrich) supplemented RPMI-1640 (Gibco) for $30 \mathrm{~min}$ at $37^{\circ} \mathrm{C}$. After incubation, tumor cells were passed through $70-\mu \mathrm{m}$ filters to remove the undigested tumor. Then, these single tumor cell suspensions were washed with ice-cold PBS with $2 \%$ FBS and stained with Live/Dead (1:1000, Invitrogen) combined with antibodies for $30 \mathrm{~min}$ at $4{ }^{\circ} \mathrm{C}$. BD LSRFortessa X-20 was used for data acquisition and FlowJo (10.4) was used for data analysis.

Western blot. Cell pellets were lysed by RIPA buffer (Sigma, R0278) with a complete EDTA-free protease inhibitor cocktail (Roche, 11836170001). Protein concentrations were quantified with the Pierce BCA Protein Assay Kit (Thermo Fisher). Equal amounts of protein $(10-20 \mu \mathrm{g})$ were run with SDS-PAGE for separation on $4-12 \%$ Bis-Tris NuPage gels or 3-8\% Tris-Acetate NuPage gels (Thermo Fisher). Protein was transferred to $0.45 \mu \mathrm{m}$ PVDF membranes (Merck) 
Membranes were blocked in Tris-buffered saline plus $0.1 \%$ Tween 20 (TBST) dissolved $5 \%$ non-fat dry milk for $1 \mathrm{~h}$ at room temperature followed by overnight incubation with primary antibodies at $4{ }^{\circ} \mathrm{C}$. Membranes were washed by TBST and incubated with HRP-conjugated secondary antibodies for $1 \mathrm{~h}$ at room temperature. HRP was activated with Supersignal West Dura Extended Duration Substrate (Pierce) and visualized with ChemiDoc Imaging System (Bio-Rad).

Phos-tag SDS-PAGE electrophoresis. To measure phosphorylation of AGO2, SDS-PAGE gels (7\%) were supplemented with Phos-tag AAL solution (Wako) according to the manufacturer's recommendations. Gels after SDS-PAGE running were incubated in transfer buffer supplemented with $1 \mathrm{mM}$ EDTA for $20 \mathrm{~min}$, and then soaked in normal transfer buffer for $10 \mathrm{~min}$. Proteins were transferred to a nitrocellulose membrane and standard western blotting procedures were subsequently followed.

Antibodies and compounds. For western blot, primary antibodies against mouse ANKRD52 (Santa Cruz, sc-398544) and CK1a (Santa Cruz, sc-6477) were used with dilution as 1:100; human ANKRD52 (Bethyl, A302-372A), OVA (Sigma, SAB5300165), $\beta$-Actin (CST, 4967), JAK1 (CST, 3332), p-STAT1 (Tyr701) (CST, 9167), p-STAT3 (Tyr705) (CST, 9145), SOCS3 (CST, 52113), TAP1 (CST, 12341), FLAG (Sigma, F3165), Vinculin (Sigma, v9131), PPP6C (Abcam, EPR8764), AGO2 (CST, 2897 S), XPO5 (CST, 12565 S), and DGCR8 (Abcam, ab191875; Proteintech, 10996-1-AP) were used with dilution as 1:1000. Londaysin (S657801) purchased from Selleck Chem were dissolved in DMSO (Thermo Fisher).

For flow cytometry, the following anti-mouse fluorochrome-conjugated antibodies were used with dilution as 1:100: AF700 anti-CD45 (560510), PerCPCy5.5 anti-CD4 (550954), BV510 anti-CD8 (563068) and BV605 anti-CD44 (563058), BV605 anti-CD25 (563061) purchased from BD Biosciences; PE anti$\mathrm{H} 2 \mathrm{~K}^{\mathrm{b}} / \mathrm{H} 2 \mathrm{D}^{\mathrm{b}}$ (114607), APC anti-H2K $\mathrm{K}^{\mathrm{b}}$ bound to SIINFEKL (141605), FITC antiLag-3 (369308), FITC anti-CD69 (104506) and AF594 anti-GZMB (372216) purchased from BioLegend; PerCP-Cy5.5 anti-CD274 (46-5982-82), PE-Cy7 antiFoxP3 (25-5773-82) and PE anti-Tim-3 (12-5870-82) purchased from ebioscience.

Antigen presentation analysis. OVA-treated ( $1 \mu \mathrm{M}$ for $2 \mathrm{~h}) \mathrm{MC} 38$ cells were simulated with $10 \mathrm{ng} / \mathrm{ml}$ IFN $\gamma$ (CST, 39127) for $24 \mathrm{~h}$. Then tumor cells were trypsinized and washed in PBS $+2 \%$ FBS, stained with antibodies against cell surface $\mathrm{H}_{2} \mathrm{~K}^{\mathrm{b}}$ and $\mathrm{H} 2 \mathrm{~K}^{\mathrm{b}}$ bound to SIINFEKL as the manufacturer's instructions and then analyzed on an BD LSRFortessa X-20 flow cytometry system. FlowJo (10.4) was used for data analysis.

Quantitative RT-PCR. Total RNA was isolated with TRIzol Reagent (invitrogen, 15596026) from tumor cells as Invitrogen user guide. cDNAs were synthesized from $1 \mu \mathrm{g}$ of total RNA using the SPARKscritpt II RT Plus Kit (SparkJade) and were amplified by $2 \times$ SYBR Green qPCR Mix (SparkJade) using Quantstudio 7 Real-Time PCR System (Life Technologies) according to the manufacturer's protocols. Relative mRNA expression was evaluated after normalization for Gapdh expression. Each experiment was performed in triplicate. The primers used for quantitative RT-PCR are listed in Supplementary Data 5.

Luciferase reporter assay. psiCheck 2 luciferase reporter plasmids (Promega) containing WT or mutated SOCS1 $3^{\prime}$ UTR and miR155-expressing or empty pMDH-PGK-EGFP plasmids were gifts from Dr. Li-Fan Lu at UCSD ${ }^{48}$. These plasmids were co-transfected into $293 \mathrm{~T}$ cells (which were seeded at $5 \times 10^{5}$ cells/ well in 6-well plates 1 day prior to transfection) using Lipofectamine 3000 reagent (Invitrogen). Cells were harvested $24 \mathrm{~h}$ later, and luciferase activity was assessed with the Dual-Luciferase Reporter Assay System (Promega) according to the manufacturer's protocol

TCGA dataset analysis. The somatic mutation data inferred by MuTect2, mRNA expression (FPKM) and clinical survival data for 33 TCGA cohorts were downloaded from UCSC Xena (GDC TCGA, https://gdc.xenahubs.net) with R package UCSCXenaTools ${ }^{75}$. The Spearman correlation between ANKRD52 or miRNA machinery expression and SOCS1 in TCGA dataset was calculated by TIMER ${ }^{49}$. The Spearman correlation between ANKRD52 or miRNA machinery expression and $\mathrm{T}$ cell infiltration in TCGA dataset was calculated by $\mathrm{EPIC}^{76}$, which were reported as more suitable for $\mathrm{CD}^{+}$and $\mathrm{CD}^{+} \mathrm{T}$ cell infiltration analysis ${ }^{77}$. The impact of mutations in miRNA machinery on survival was shown for patients whose tumors had higher $(>75 \%)$ or lower $(<75 \%)$ infiltration score of cytotoxic CD8 T cells (mean of CD8A, CD8B, GZMB, and PRF1) ${ }^{9}$. All of TCGA analysis data (containing the results of TIMER and EPIC analysis) were obtained from TIMER2.0 database ${ }^{49}$ (http://timer.cistrome.org).

Statistical analysis. For all experiments, the number of technical and/or biological replicates is provided in the figure legends or text. Microsoft Excel (16.27) was used to organize data into tables. In all cases, $* * * * P<0.0001, * * * P<0.001, * * P<0.01$, ${ }^{*} P<0.05$. Statistical analyses were performed using Graph-Pad Prism (8.2.1) or the $R$ language (3.6.0) programming environment using RStudio (1.2.1335) to calculate the " $p$-value". In CRISPR screen or RNA-Seq analysis, the expression matrix is modeled using an overdispersed poisson model in EdgeR after above-mentioned normalization. Gene dispersions are then estimated by conditional maximum likelihood and shrunk using an empirical Bayes procedure. Finally, differential expression is assessed using an adapted Fisher's exact test and get the ' $p$-value"74 In MAGeCK RRA software, just like in EdgeR, the " $p$-value" is calculated by randomly permuting sgRNA labels. A negative binomial (NB) model is used to tes whether sgRNA abundance differs significantly between treatments and controls and a modified robust ranking aggregation (RRA) algorithm is used to identify positively or negatively selected genes based on $p$-value calculated from the NB model $^{73}$. In GSEA, the " $p$-value" is calculated by the permutation test. All 'Spearman's correlation' with ' $p$-value” data were obtained from Depmap or TIMER 2.0 database as mentioned above.

Reporting Summary. Further information on research design is available in the Nature Research Reporting Summary linked to this article.

\section{Data availability}

The raw FASTQ files and the source datasets generated and analyzed in this study for the sequencing data are available in the Genome Sequence Archive (GSA) database with accession number CRA004140, CRA004141, CRA004145, CRA004146). Descriptions of the analyses, tools and algorithms are provided in the Methods or GSA. The TCGA mutation data and analysis data used in this study are available in the UCSC Xena database and TIMER2.0 database, respectively. The remaining data are available within the Article, Supplementary Information or Source Data file. Source data are provided with this paper.

Received: 25 April 2021; Accepted: 15 November 2021; Published online: 01 December 2021

\section{References}

1. Sharma, P., Hu-Lieskovan, S., Wargo, J. A. \& Ribas, A. Primary, adaptive, and acquired resistance to cancer immunotherapy. Cell. 168, 707-723 (2017).

2. Havel, J. J., Chowell, D. \& Chan, T. A. The evolving landscape of biomarkers for checkpoint inhibitor immunotherapy. Nat. Rev. Cancer. 19, 133-150 (2019).

3. Hegde, P. S. \& Chen, D. S. Top 10 challenges in cancer immunotherapy. Immunity. 52, 17-35 (2020).

4. Zaretsky, J. M. et al. Mutations associated with acquired resistance to PD-1 blockade in melanoma. N. Engl. J. Med. 375, 819-829 (2016).

5. Miao, D. et al. Genomic correlates of response to immune checkpoint therapies in clear cell renal cell carcinoma. Science. 359, 801-806 (2018).

6. Hugo, W. et al. Genomic and transcriptomic features of response to anti-PD-1 therapy in metastatic melanoma. Cell. 165, 35-44 (2016).

7. Galon, J. \& Bruni, D. Tumor immunology and tumor evolution: intertwined histories. Immunity. 52, 55-81 (2020).

8. Buisson, R. et al. Passenger hotspot mutations in cancer driven by APOBEC3A and mesoscale genomic features. Science. 364 https://doi.org/10.1126/ science.aaw2872 (2019).

9. Pan, D. et al. A major chromatin regulator determines resistance of tumor cells to T cell-mediated killing. Science. 359, 770-77 (2018). https://doi.org/ 10.1126/science.aao1710.

10. Lawson, K. A. et al. Functional genomic landscape of cancer-intrinsic evasion of killing by T cells. Nature. https://doi.org/10.1038/s41586-020-2746-2 (2020)

11. Manguso, R. T. et al. In vivo CRISPR screening identifies Ptpn 2 as a cancer immunotherapy target. Nature. 547, 413-418 (2017).

12. Wang, G. et al. CRISPR-GEMM pooled mutagenic screening identifies KMT2D as a major modulator of immune checkpoint blockade. Cancer Discov. https://doi.org/10.1158/2159-8290.CD-19-1448 (2020).

13. Sade-Feldman, M. et al. Resistance to checkpoint blockade therapy through inactivation of antigen presentation. Nat. Commun. 8, 1136 (2017).

14. Sucker, A. et al. Acquired IFNgamma resistance impairs anti-tumor immunity and gives rise to T-cell-resistant melanoma lesions. Nat. Commun. 8, 15440 (2017).

15. Patel, S. J. et al. Identification of essential genes for cancer immunotherapy. Nature. 548, 537-542 (2017).

16. Ishizuka, J. J. et al. Loss of ADAR1 in tumours overcomes resistance to immune checkpoint blockade. Nature. https://doi.org/10.1038/s41586-0180768-9 (2018)

17. $\mathrm{Li}, \mathrm{F}$. et al. In vivo epigenetic CRISPR screen identifies Asfla as an immunotherapeutic target in Kras-mutant lung adenocarcinoma. Cancer Disco. 10, 270-287 (2020).

18. Vredevoogd, D. W. et al. Augmenting immunotherapy impact by lowering tumor TNF cytotoxicity threshold. Cell. https://doi.org/10.1016/ j.cell.2019.06.014 (2019). 
19. Bartel, D. P. MicroRNAs: target recognition and regulatory functions. Cell. 136, 215-233 (2009).

20. Lin, S. \& Gregory, R. I. MicroRNA biogenesis pathways in cancer. Nat. Rev. Cancer. 15, 321-333 (2015).

21. Golden, R. J. et al. An argonaute phosphorylation cycle promotes microRNAmediated silencing. Nature. 542, 197-202 (2017).

22. Wang, D. et al. Uncovering the cellular capacity for intensive and specific feedback self-control of the argonautes and MicroRNA targeting activity. Nucleic Acids Res. 48, 4681-4697 (2020)

23. Hill, D. A. et al. DICER1 mutations in familial pleuropulmonary blastoma. Science. 325, 965 (2009).

24. Heravi-Moussavi, A. et al. Recurrent somatic DICER1 mutations in nonepithelial ovarian cancers. N. Engl. J. Med. 366, 234-242 (2012).

25. Foulkes, W. D., Priest, J. R. \& Duchaine, T. F. DICER1: mutations, microRNAs and mechanisms. Nat. Rev. Cancer. 14, 662-672 (2014).

26. Walz, A. L. et al. Recurrent DGCR8, DROSHA, and SIX homeodomain mutations in favorable histology Wilms tumors. Cancer Cell. 27, 286-297 (2015).

27. Wegert, J. et al. Mutations in the SIX1/2 pathway and the DROSHA/DGCR8 miRNA microprocessor complex underlie high-risk blastemal type Wilms tumors. Cancer Cell. 27, 298-311 (2015).

28. Hodis, E. et al. A landscape of driver mutations in melanoma. Cell. 150, 251-263 (2012).

29. Kumar, M. S., Lu, J., Mercer, K. L., Golub, T. R. \& Jacks, T. Impaired microRNA processing enhances cellular transformation and tumorigenesis. Nat. Genet. 39, 673-677 (2007).

30. Merritt, W. M. et al. Dicer, Drosha, and outcomes in patients with ovarian cancer. N. Engl. J. Med. 359, 2641-2650 (2008).

31. Lin, R. J. et al. microRNA signature and expression of Dicer and Drosha can predict prognosis and delineate risk groups in neuroblastoma. Cancer Res. 70, 7841-7850 (2010).

32. Chen, S. et al. Genome-wide CRISPR screen in a mouse model of tumor growth and metastasis. Cell. 160, 1246-1260 (2015).

33. Maire, C. L. et al. Glioma escape signature and clonal development under immune pressure. J. Clin. Invest. https://doi.org/10.1172/JCI138760 (2020).

34. Ghandi, M. et al. Next-generation characterization of the cancer cell line encyclopedia. Nature. 569, 503-508 (2019).

35. Thorsson, V. et al. The immune landscape of cancer. Immunity. 48, 812-830 e814 (2018).

36. McKenna, A. et al. The Genome Analysis Toolkit: a MapReduce framework for analyzing next-generation DNA sequencing data. Genome Res. 20, 1297-1303 (2010).

37. Yizhak, K. et al. RNA sequence analysis reveals macroscopic somatic clonal expansion across normal tissues. Science. $\mathbf{3 6 4} \mathrm{https} / / /$ doi.org/10.1126/ science.aaw0726 (2019).

38. Sharpe, A. H. \& Pauken, K. E. The diverse functions of the PD1 inhibitory pathway. Nat. Rev. Immunol. 18, 153-167 (2018).

39. Shin, D. S. et al. Primary resistance to PD-1 blockade mediated by JAK1/2 mutations. Cancer Disco. 7, 188-201 (2017).

40. Williams, J. B. et al. Tumor heterogeneity and clonal cooperation influence the immune selection of IFN-gamma-signaling mutant cancer cells. Nat. Commun. 11, 602 (2020).

41. Benci, J. L. et al. Opposing functions of interferon coordinate adaptive and innate immune responses to cancer immune checkpoint blockade. Cell. 178, 933-948 e914 (2019).

42. Nishimura, H. \& Honjo, T. PD-1: an inhibitory immunoreceptor involved in peripheral tolerance. Trends Immunol. 22, 265-268 (2001).

43. Refaeli, Y., Van Parijs, L., Alexander, S. I. \& Abbas, A. K. Interferon gamma is required for activation-induced death of T lymphocytes. J. Exp. Med. 196, 999-1005 (2002).

44. Humblin, E. \& Kamphorst, A. O. CXCR3-CXCL9: It's All in the Tumor. Immunity. 50, 1347-1349 (2019).

45. Villarino, A. V., Kanno, Y. \& O'Shea, J. J. Mechanisms and consequences of Jak-STAT signaling in the immune system. Nat. Immunol. 18, 374-384 (2017).

46. Van Allen, E. M. et al. Genomic correlates of response to CTLA-4 blockade in metastatic melanoma. Science. 350, 207-211 (2015).

47. Riaz, N. et al. Tumor and microenvironment evolution during immunotherapy with nivolumab. Cell. 171, 934-949 e916 (2017).

48. Lu, L. F. et al. Foxp3-dependent microRNA155 confers competitive fitness to regulatory $\mathrm{T}$ cells by targeting SOCS1 protein. Immunity. 30, 80-91 (2009).

49. Li, T. et al. TIMER2.0 for analysis of tumor-infiltrating immune cells. Nucleic Acids Res. 48, W509-W514 (2020).

50. Guinney, J. et al. The consensus molecular subtypes of colorectal cancer. Nat. Med. 21, 1350-1356 (2015).

51. Sveen, A. et al. Colorectal cancer consensus molecular subtypes translated to preclinical models uncover potentially targetable cancer cell dependencies. Clin. Cancer Res. 24, 794-806 (2018).
52. Anzalone, A. V., Koblan, L. W. \& Liu, D. R. Genome editing with CRISPR-Cas nucleases, base editors, transposases and prime editors. Nat. Biotechnol. 38, 824-844 (2020).

53. Hayashi, K. et al. Abrogation of protein phosphatase 6 promotes skin carcinogenesis induced by DMBA. Oncogene. 34, 4647-4655 (2015).

54. Kumar, M. S. et al. Dicer1 functions as a haploinsufficient tumor suppressor. Genes Dev. 23, 2700-2704 (2009).

55. Melo, S. A. et al. A genetic defect in exportin-5 traps precursor microRNAs in the nucleus of cancer cells. Cancer Cell. 18, 303-315 (2010).

56. Leaderer, D. et al. Genetic and epigenetic association studies suggest a role of microRNA biogenesis gene exportin-5 (XPO5) in breast tumorigenesis. Int J. Mol. Epidemiol. Genet. 2, 9-18 (2011).

57. Litchfield, K. et al. Meta-analysis of tumor- and T cell-intrinsic mechanisms of sensitization to checkpoint inhibition. Cell 184, 596-614 e514 (2021).

58. Baer, C. et al. Suppression of microRNA activity amplifies IFN-gammainduced macrophage activation and promotes anti-tumour immunity. Nat. Cell Biol. 18, 790-802 (2016).

59. Gurung, C. et al. Dicer represses the interferon response and the doublestranded RNA-activated protein kinase pathway in mouse embryonic stem cells. J. Biol. Chem. 296, 100264 (2021).

60. Gocher, A. M., Workman, C. J. \& Vignali, D. A. A. Interferon-gamma: teammate or opponent in the tumour microenvironment? Nature reviews. Immunology. https://doi.org/10.1038/s41577-021-00566-3 (2021).

61. Cortez, M. A. et al. Role of miRNAs in immune responses and immunotherapy in cancer. Genes, Chromosomes Cancer 58, 244-253 (2019).

62. Kronke, J. et al. Lenalidomide induces ubiquitination and degradation of CK1alpha in del(5q) MDS. Nature. 523, 183-188 (2015).

63. Gorgun, G. et al. Lenalidomide enhances immune checkpoint blockadeinduced immune response in multiple myeloma. Clin. Cancer Res. 21, 4607-4618 (2015)

64. Dobin, A. et al. STAR: ultrafast universal RNA-seq aligner. Bioinformatics. 29, 15-21 (2013).

65. Li, B. \& Dewey, C. N. RSEM: accurate transcript quantification from RNA-Sec data with or without a reference genome. BMC Bioinforma. 12, 323 (2011).

66. McCarthy, D. J., Chen, Y. \& Smyth, G. K. Differential expression analysis of multifactor RNA-Seq experiments with respect to biological variation. Nucleic Acids Res. 40, 4288-4297 (2012).

67. Falcon, S. \& Gentleman, R. Using GOstats to test gene lists for GO term association. Bioinformatics. 23, 257-258 (2007).

68. Liberzon, A. et al. The molecular signatures database (MSigDB) hallmark gene set collection. Cell Syst. 1, 417-425 (2015)

69. Subramanian, A. et al. Gene set enrichment analysis: a knowledge-based approach for interpreting genome-wide expression profiles. Proc. Natl Acad. Sci. 102, 15545-15550 (2005)

70. Petitprez, F. et al. The murine Microenvironment Cell Population counter method to estimate abundance of tissue-infiltrating immune and stromal cell populations in murine samples using gene expression. Genome Med. 12, 86 (2020).

71. Wang, K., Li, M. \& Hakonarson, H. ANNOVAR: functional annotation of genetic variants from high-throughput sequencing data. Nucleic Acids Res. 38, e164 (2010).

72. Joung, J. et al. Genome-scale CRISPR-Cas 9 knockout and transcriptional activation screening. Nat. Protoc. 12, 828-863 (2017).

73. Wang, B. et al. Integrative analysis of pooled CRISPR genetic screens using MAGeCKFlute. Nat. Protoc. 14, 756-780 (2019).

74. Dai, Z. et al. edgeR: a versatile tool for the analysis of shRNA-seq and CRISPR-Cas9 genetic screens. F1000Res. 3, 95 (2014).

75. Wang, S. \& Liu, X. The UCSCXenaTools R package: a toolkit for accessing genomics data from UCSC Xena platform, from cancer multi-omics to singlecell RNA-seq. J. Open Source Softw. 4, https://doi.org/10.21105/joss.01627 (2019).

76. Rosenthal, R. et al. Neoantigen-directed immune escape in lung cancer evolution. Nature. 567, 479-485 (2019).

77. Sturm, G. et al. Comprehensive evaluation of transcriptome-based cell-type quantification methods for immuno-oncology. Bioinformatics. 35, i436-i445 (2019).

\section{Acknowledgements}

We thank all members of the Y. Cang laboratory; H. P. Wang at ShanghaiTech for immunological suggestions; L.Y. Zhang at SHTU, X. X. Xie, and Q. Qian at WuXi for bioinformatic suggestions; B. Sun at SIBCB for discussions and the gift of OT-I mice; L. F. Lu at UCSD for assistance with luciferase reporter assay; Y. N. Li at ZJU for coloring the mouse in the schematic; and the Molecular and Cell Biology Core Facility (MCBCF), the Molecular Imaging Core Facility (MICF) and the Multi-Omics Core Facility (MOCF) at the School of Life Science and Technology, ShanghaiTech for providing technical support. T. Y. Song was supported by Shanghai Post-doctoral Excellence Program from Shanghai Municipal Human Resources and Social Security Bureau. This research was funded by 2019M651613 (T. Y. Song) and 2020T130669 (T. Y. Song) from China 
Postdoctoral Science Foundation; 82003803 (T. Y. Song), 92053118 and 31970671 (Y. Cang) from National Natural Science Foundation of China; and 18JC1413900 (Y. Cang) from Shanghai Science and Technology Committee.

\section{Author contributions}

T. Y. S and Y. C. conceived and planned the study. T. Y. S. and H. X. Z performed CRISPR screens. T. Y. S., M. L., H. J. F., M. W. Z., and Y. L. performed the animal studies T. Y. S, M. W. Z., H. J. F., Y. L., C. L. G., H. X. Z, and M. L. performed the molecular and cell-based experiments. T. Y. S., M. L., H. X. Z., C. L. G., W. R. Z., B. P., M. W. Z., H. J. F., Y. L., and Z. G. P. analyzed the data. M. F. S., Y. L. Y., M. L., T. Y. S., and D. W. H. did all the bioinformatic analysis. T. Y. S. and Y. C. wrote the manuscript, M. L. and H. X. Z. proofread it, and all other authors contributed to the revisions. H. J. F. and M. W. Z. contributed equally to this study.

\section{Competing interests}

The authors declare no competing interests.

\section{Additional information}

Supplementary information The online version contains supplementary material available at https://doi.org/10.1038/s41467-021-27331-3.

Correspondence and requests for materials should be addressed to Yong Cang.
Peer review information Nature Communications thanks the anonymous reviewer(s) for their contribution to the peer review of this work.

Reprints and permission information is available at http://www.nature.com/reprints

Publisher's note Springer Nature remains neutral with regard to jurisdictional claims in published maps and institutional affiliations.

(c) Open Access This article is licensed under a Creative Commons Attribution 4.0 International License, which permits use, sharing, adaptation, distribution and reproduction in any medium or format, as long as you give appropriate credit to the original author(s) and the source, provide a link to the Creative Commons license, and indicate if changes were made. The images or other third party material in this article are included in the article's Creative Commons license, unless indicated otherwise in a credit line to the material. If material is not included in the article's Creative Commons license and your intended use is not permitted by statutory regulation or exceeds the permitted use, you will need to obtain permission directly from the copyright holder. To view a copy of this license, visit http://creativecommons.org/ licenses/by/4.0/.

(C) The Author(s) 2021 